\title{
Extracellular matrix fiber microarchitecture is region-specific in bicuspid aortic valve-associated ascending aortopathy
}

\author{
Alkiviadis Tsamis, PhD, ${ }^{\mathrm{a}, \mathrm{b}, \mathrm{c}}$ Julie A. Phillippi, PhD, ${ }^{\mathrm{a}, \mathrm{b}, \mathrm{c}, \mathrm{d}}$ Ryan G. Koch, BSc, ${ }^{\mathrm{a}}$ Patrick G. Chan, MD, \\ Jeffrey T. Krawiec, BSc, ${ }^{\text {a,b,c }}$ Antonio D'Amore, PhD,, a,c,e,f,g Simon C. Watkins, PhD, ${ }^{\text {h }}$ \\ William R. Wagner, PhD, ${ }^{\mathrm{a}, \mathrm{b}, \mathrm{c}, \mathrm{e}}$ David A. Vorp, PhD, ${ }^{\mathrm{a}, \mathrm{b}, \mathrm{c}, \mathrm{d}, \mathrm{e}}$ and Thomas G. Gleason, MD ${ }^{\mathrm{a}, \mathrm{b}, \mathrm{c}, \mathrm{d}}$
}

\begin{abstract}
Objective: Ascending thoracic aortic aneurysm (ATAA) in patients with bicuspid aortic valve (BAV) commonly dilate asymmetrically compared with patients with tricuspid aortic valve (TAV). This discrepancy in aneurysm geometry led us to hypothesize that microarchitectural differences underlie the observed asymmetric dilatation pattern. The purpose of this study was to characterize the microarchitectural distinctions of the extracellular matrix of the 2 phenotypes with a focus on the proportion of radially oriented elastin and collagen fibers in different circumferential aortic regions.
\end{abstract}

Methods: Aortic tissue rings were obtained just distal to the sinotubular junction from patients with BAV or TAV undergoing elective aneurysm repair. They were sectioned into three circumferentially based regions according to adjacent aortic sinus segment (left coronary sinus [L], right coronary sinus [R], or noncoronary sinus $[\mathrm{N}])$. Multiphoton microscopy was used to quantify and characterize the number of radially oriented elastin and collagen fibers.

Results: There were fewer radially oriented fibers in medial region $\mathrm{N}$ and medialintimal region $\mathrm{R}$ of BAV-ATAAs when compared with TAV-ATAAs (medial region $\mathrm{N}$, amplitude of angular undulation of elastin $=10.67^{\circ} \pm 1.35^{\circ}$ vs $15.58^{\circ} \pm 1.91^{\circ} ; P=.041$; medial-intimal region $\mathrm{R}$, amplitude of angular undulation of elastin $=9.83^{\circ} \pm 0.83^{\circ}$ vs $\left.14.72^{\circ} \pm 1.64^{\circ} ; P=.015\right)$. Conversely, fibers became more radially oriented in the medial-intimal region L of BAV-ATAA when compared with TAV-ATAA (amplitude of angular undulation of collagen $=18.67^{\circ} \pm 0.95^{\circ}$ vs $\left.14.56^{\circ} \pm 1.37^{\circ} ; P=.041\right)$.

Conclusions: The differential pattern of fiber orientation noted between $\mathrm{L}$ and $\mathrm{N}-\mathrm{R}$ regions help explain the unique pattern of greater curvature dilatation of BAVATAA. The distinctions noted in matrix microarchitecture may form the basis of differing aneurysm geometries and aortic wall integrities in ATAAs arising in these different valve morphologies. (J Thorac Cardiovasc Surg 2016;151:1718-28)

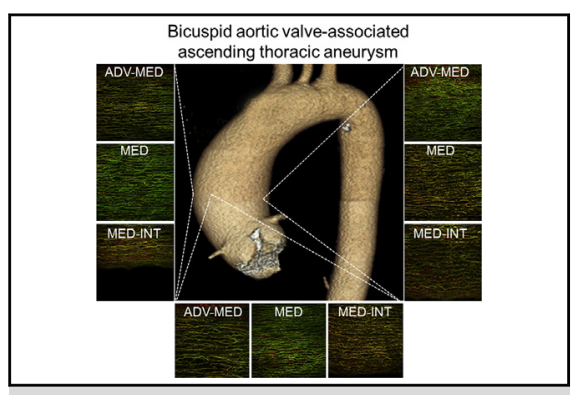

Regional abnormalities in elastin fiber microarchitecture in bicuspid aortic valve-associated aneurysms. ADV-MED, the media near the adventitial border; MED, the mid portion relative to the radial plane of the media; INT-MED, the media near the intimal border.

\section{Central Message}

Bicuspid valve-associated asymmetric aortic dilatation correlates with region-specific changes in elastin fiber microarchitecture.

\section{Perspective}

Multiphoton microscopy was employed to characterize the microarchitecture of ascending aortic aneurysms from bicuspid (BAV) and tricuspid aortic valve patients. Region-specific decrease in elastin fiber radial orientation was found in BAV-associated aneurysms. The findings offer an explanation for the asymmetric dilatation pattern of BAV-aneurysms toward the greater curvature of aorta.

See Editorial Commentary page 1728.
From the ${ }^{\mathrm{a}}$ Departments of Bioengineering, ${ }^{\mathrm{d}}$ Cardiothoracic Surgery, and ${ }^{\mathrm{e}}$ Surgery, ${ }^{\mathrm{b}}$ Center for Vascular Remodeling and Regeneration, ${ }^{\mathrm{h}}$ Center for Biologic Imaging, and ${ }^{\mathrm{c}} \mathrm{McG}$ cowan Institute for Regenerative Medicine, University of Pittsburgh, Pittsburgh, Pa; and ${ }^{\mathrm{f} F o n d a z i o n e ~ R i . M E D, ~ a n d ~}{ }^{\mathrm{g}}$ Department of Chemical, Management, Computer and Mechanical Engineering (DICIGIM), University of Palermo, Palermo, Italy.

Supported by the Swiss National Science Foundation Fellowships for Advanced Researchers (Nos. PA00P2_139684 and PA00P2_145399) (to A.T.), by the University of Pittsburgh Swanson School of Engineering (to R.G.K.), by the Fondazione Ri.MED (to A.D.A.), by National Institutes of Health grant No. R01 HL109132 (to T.G.G., J.A.P., and D.A.V.), and by the University of Pittsburgh Department of Cardiothoracic Surgery (to T.G.G. and D.A.V.).

Received for publication Jan 28, 2015; revisions received Jan 25, 2016; accepted for publication Feb 7, 2016; available ahead of print March 12, 2016.

Address for reprints: Thomas G. Gleason, MD, Division of Cardiac Surgery, Department of Cardiothoracic Surgery, University of Pittsburgh, 5200 Centre Ave, Ste 715, Pittsburgh, PA 15232 (E-mail: gleasontg@upmc.edu).

$0022-5223 / \$ 36.00$

Copyright (c) 2016 by The American Association for Thoracic Surgery

http://dx.doi.org/10.1016/j.jtcvs.2016.02.019
Bicuspid aortic valve (BAV) is the most common congenital heart defect, arising in $1 \%$ to $2 \%$ of the general population, and along with predisposing to valvular dysfunction the defect is frequently associated with the development of ascending aortopathy and aneurysm. ${ }^{1-5}$ Patients with BAV uniformly have a large-diameter ascending aorta, indicative of the aortopathy, compared with age- and sex-matched controls. ${ }^{6}$ Consequently, patients with BAV are at

Scanning this QR code will take you to supplemental figures for this article. 


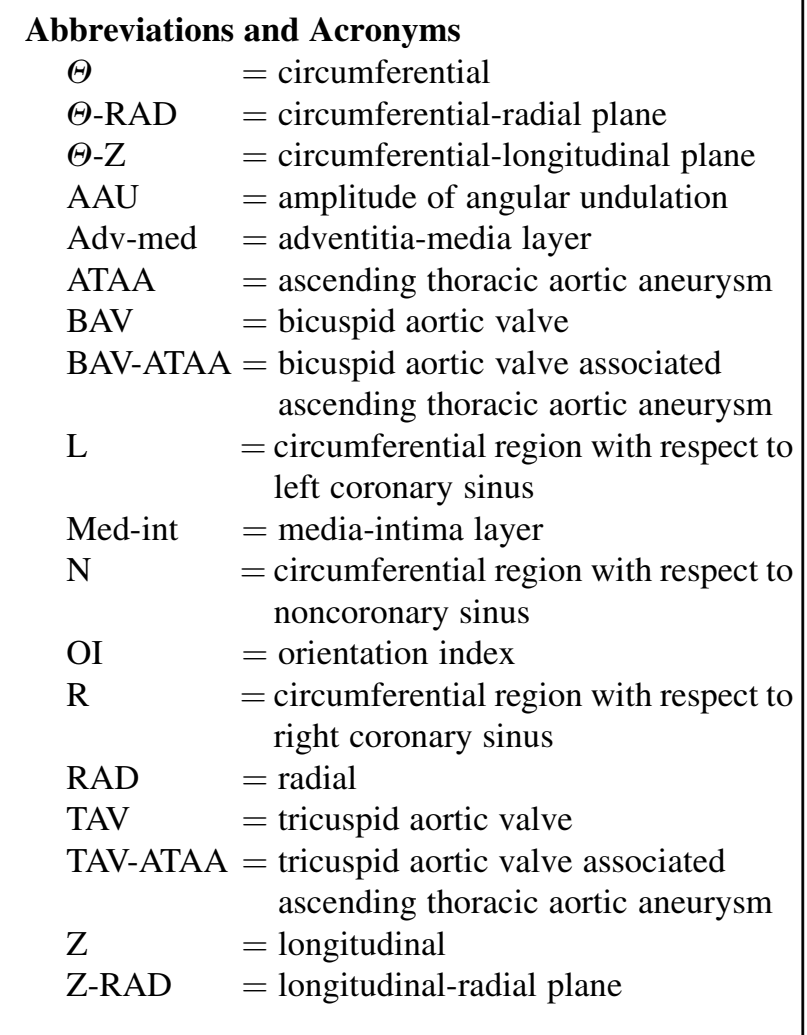

significant risk of aortic dissection. ${ }^{5,7-9}$ There is a bimodal age distribution among ascending thoracic aortic aneurysms (ATAAs), with patients with BAV presenting an average of 10 to 15 years earlier than the degenerative ATAAs that form in patients with tricuspid aortic valve (TAV). ${ }^{4}$ Furthermore, many BAV-associated ATAAs tend to dilate asymmetrically toward the greater curvature of the ascending aorta (extending from the right and noncoronary sinuses) compared with a more symmetric dilatation pattern that occurs in patients with TAV. ${ }^{10-13}$

ATAAs rupture or dissect when blood pressure-induced stresses exceed the tensile strength of the aortic wall. We have identified distinct differences in the aortic wall tensile strength in ATAAs from BAV versus TAV patients. ${ }^{14}$ The tensile strength of BAV-ATAAs was found to be higher when compared with TAV-ATAAs in both the circumferential $(\Theta)$ and longitudinal $(Z)$ directions, and tensile strength in the $\Theta$ direction was higher than in $\mathrm{Z}$ direction for both BAV-ATAAs and TAV-ATAAs. The delamination strength (ie, resistance to separation between aortic layers, analogous to clinical dissection) of BAV-ATAA and TAV-ATAA specimens each had lower delamination strength when compared with specimens from nonaneurysmal aortas in either $\mathrm{Z}$ or $\Theta$ direction, and BAV-ATAA specimens had lower delamination strength than TAV-ATAA specimens, suggesting that the former may have an increased propensity for aortic dissection. ${ }^{15}$

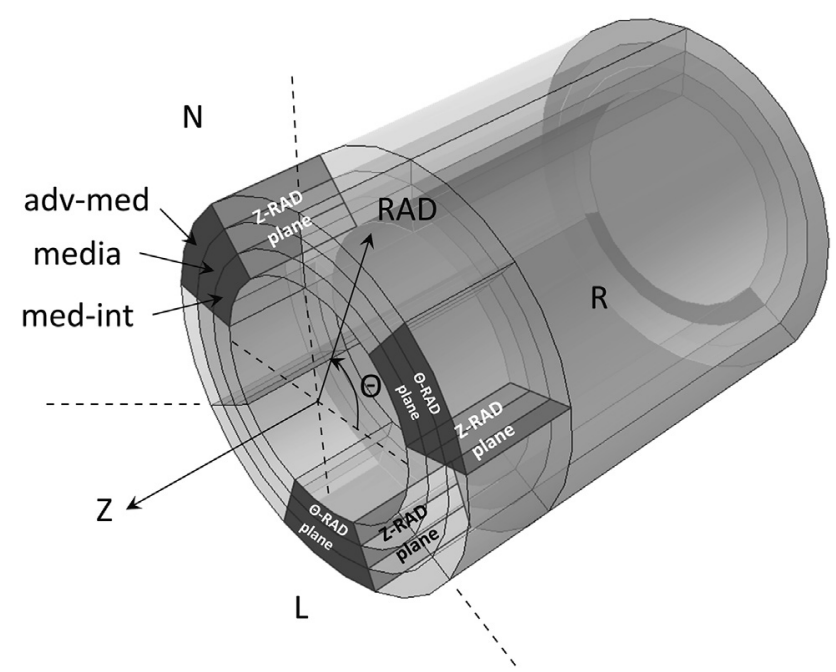

FIGURE 1. Rings of ascending thoracic aortic aneurysm $(A T A A)$ were cut into 3 circumferential $(\Theta)$ regions according to their anatomic location with respect to the left coronary sinus $(L)$, right coronary sinus $(R)$, and noncoronary sinus $(N)$, respectively. The grey-shaded areas in each of the $3 \Theta$-regions indicate the $\Theta$-radial (RAD) and longitudinal (Z) RAD planes across the aortic wall thickness, which were the imaging planes of the microscopy analysis of the study. After fixing these samples, multiphoton microscopy images were acquired in 3 locations across the wall thickness; that is, adventitiamedia ( $a d v$-med), media, and media-intima (med-int), in both $\Theta$-RAD and Z-RAD planes (in total 18 wall locations for each ATAA phenotype). $Z-R A D$, Longitudinal-radial plane; $\Theta-R A D$, circumferential-radial plane.

The strength of the ascending aortic wall may be diminished by the locally disrupted microarchitecture in the presence of aneurysm, mainly characterized by the transmural content and orientation of both elastin and collagen fibers. ${ }^{16}$ The interlaminar elastin and collagen fibers and particularly those fibers that are radially (RAD) oriented, which are the fibers that bear the maximum contribution of radial forces, contribute to the adhesive cell-matrix forces that hold the aortic layers together under applied hemodynamic loading conditions. We are the first group to assume that reorganization of interlaminar elastin and collagen fibers that leads to a reduction in the quantity of RAD components of these fibers could decrease the medial delamination strength in the degenerated ATAA wall ${ }^{15}$ and could lead to spontaneous tissue tears when the pressure-induced wall stresses exceed this strength. It has also been reported that the microarchitecture during aortic dissection is characterized by a loss of elastin content and a decrease in interlaminar elastin fibers. ${ }^{17}$ Furthermore, these fibers are fragmented and irregularly arranged, ${ }^{16,18,19}$ and are accompanied by cystic medial degeneration. ${ }^{19,20}$ Although we detected no difference in total collagen content between BAV-ATAA and TAV-ATAA,${ }^{14}$ scanning electron microscopic analyses of the aortic media en face (ie, $\Theta-Z$ plane) revealed architectural differences at the delamination plane between TAV versus BAV-ATAA, which supports the notion that 
TABLE 1. Clinical data of patients for aortic tissue used for multiphoton microscopy

\begin{tabular}{|c|c|c|c|c|c|}
\hline All samples & $\mathbf{N}(\mathbf{M} / \mathbf{F})$ & $\begin{array}{l}\text { Bicuspid aortic } \\
\text { valve } \\
\text { morphotype (n) } \\
\end{array}$ & $\begin{array}{c}\text { Age }(y) \\
\text { (median } \pm \text { standard } \\
\text { deviation) }\end{array}$ & $\begin{array}{c}\text { Aortic diameter }(\mathrm{mm}) \\
(\text { mean } \pm \text { standard } \\
\text { deviation })\end{array}$ & Hypertension (n) \\
\hline Tricuspid aortic valve ascending aortic aneurysm & $6(4 / 2)$ & N/A & $73 \pm 10.0$ & $54.8 \pm 6.2^{\mathrm{ns}}$ & $50 \%(3 / 6)$ \\
\hline Bicuspid aortic valve ascending aortic aneurysm & $6(6 / 0)$ & $\begin{array}{l}\text { Type } 0 \text { (3) } \\
\text { Type } 1 \text { R/L (3) }\end{array}$ & $59 \pm 15.9$ & $50.0 \pm 2.9^{\text {ns }}$ & $17 \%(1 / 6)$ \\
\hline
\end{tabular}

$M$, Male; $F$, female; $N / A=$ not available; $n s$, nonsignificant.

microstructural nuances of matrix microarchitecture of collagen and elastin may be at the heart of distinct failure modalities leading to dissection in these patient cohorts. $^{15}$

Guided by our prior observations, we recently uncovered distinct patterns of ascending aortic medial matrix remodeling in ATAA specimens from BAV when compared with TAV patients and demonstrated a more highly aligned collagen and elastin microarchitecture pattern in the $\Theta-Z$ plane $^{21}$ in accordance with our observations of increased tensile strength in BAV-ATAA when compared with TAVATAA. ${ }^{14}$ We also characterized the aortic medial microarchitecture in the longitudinal-radial (Z-RAD) and circumferential-radial $(\Theta-R A D)$ planes using multiphoton microscopy. ${ }^{22}$ We found a discrepancy in the arrangement of RAD-oriented fibers across the media of TAV-ATAA with increasing age that may be associated with the development and progression of vascular remodeling in aneurysms arising in patients with TAV.

Collectively, these data led us to hypothesize that microarchitectural differences in the $\Theta$ direction underlie the observed anatomical asymmetry of aortic dilatation based on aortic valve phenotype. To test the hypothesis, we quantified the proportion of RAD-oriented elastin and collagen fibers in different $\Theta$ regions of the human BAV-ATAA and TAV-ATAA in both Z-RAD and $\Theta$-RAD planes using a multiphoton microscopic image-based analysis.

\section{METHODS}

\section{Human Aorta Specimens}

All the collected human aortic specimens were gathered with the University of Pittsburgh's Institutional Review Board approval and with informed patient consent. Rings from nondissected ATAAs were freshly obtained from patients with either BAV $(n=6)$ or TAV $(n=6)$ with age ranging from 33 to 83 years (10 men and 2 women) undergoing elective aortic replacement at the University of Pittsburgh Medical Center due to aneurysm with or without aortic valvular replacement. The aortic rings were obtained within $2 \mathrm{~cm}$ of the sinotubular junction and cut into $3 \Theta$ regions (left coronary sinus [L], right coronary sinus [R], and noncoronary sinus $[\mathrm{N}])$ according to their anatomic location with respect to the aortic valve cusps (Figure 1). The samples were perfusion-fixed in $4 \%$ paraformaldehyde for approximately 1.5 hours. After replacement of the solution with phosphate buffered saline, the specimens were stored at $4{ }^{\circ} \mathrm{C}$ and visualized using multiphoton microscopy for evaluating the fiber microarchitecture. Table 1 summarizes patient demographic data of the aortic specimens. Patients underwent preoperative computed tomographic angiography to determine the maximal orthogonal diameter of the ascending aorta to determine their indication for aortic replacement due to aneurysm with or without the presence of aortic valvular insufficiency and/or stenosis. All patients had a maximal orthogonal aortic diameter $\geq 47 \mathrm{~mm}$.

\section{Multiphoton Microscopy}

Imaging was performed using an Olympus multiphoton microscope (Model FV10, Olympus, Center Valley, Pa) to visualize elastin and collagen fiber architecture in 18 locations of the ATAA wall, namely 3 RAD locations and three $\Theta$ regions in both Z-RAD (Figure 2) and $\Theta$-RAD planes (Figure 3) for all specimens. ${ }^{23,24}$ During imaging, the Z-RAD or $\Theta$-RAD planes of the samples faced the microscope lens to allow for visualization of RAD-oriented collagen and elastin fibers. Elastin and collagen fibers were automatically detected according to intrinsic fluorescence (channel RXD1; emission detection wavelength, $525 \pm 25 \mathrm{~nm}$ ) and second harmonic generation (channel RXD2; emission detection wavelength, $400 \pm 50 \mathrm{~nm}$ ), respectively. After acquiring images with multiphoton microscopy, the percent of fibers (elastin and collagen) oriented in the RAD direction, the average amplitude of angular undulation $(\mathrm{AAU})$ and the orientation index $(\mathrm{OI})$ of the fibers were quantified using an image-based analysis tool as outlined below.

\section{Image-Based Analysis to Characterize Wall Microarchitecture}

A custom automated image-based analysis tool developed in MATLAB (MathWorks, Inc, Natick, Mass) based on image background removal ${ }^{25}$ and on fiber detection algorithm ${ }^{26}$ was used to process the multiphoton images, revealing the collagen (Figures E1 and E2) and elastin fiber architecture (Figures E3 and E4) in both Z-RAD and $\Theta$-RAD planes of BAV-ATAA and TAV-ATAA in 3 RAD-locations within the aortic wall thickness. Multiphoton images that were captured $\leq 500 \mu \mathrm{m}$ from the outer surface of adventitia were considered to be the adventitia-media (adv-med) location. Multiphoton images that were captured $\leq 500 \mu \mathrm{m}$ from the intimal surface are referred to as the media-intima (med-int) location. Multiphoton images that were obtained between these peripheral locations (adv-med and medint) were denoted as media. If the thickness of the media was such to contain more than 1 multiphoton image $\left(0.5 \times 0.5 \mathrm{~mm}^{2}\right)$, the analysis output of these images was averaged. For an example description of the output generated by the image-based analysis tool using a multiphoton image of collagen fibers, the reader is referred to Tsamis and colleagues. ${ }^{22}$ In brief, the algorithm was able to filter the fiber components with a RAD orientation and provided the corresponding percentage of fibers oriented in the RAD direction. Also, the angle difference between the 2 peaks of the fiber angle distribution (orientation histogram) was found to be representative of fiber AAU in that plane of the tissue. A RAD-oriented fiber is defined as either an individual RAD-oriented fiber component or a RAD-oriented segment of a fiber owing to its AAU about the $\mathrm{Z}$ or $\Theta$ axis. Consequently, the percentage of fibers oriented in the RAD direction depends on 2 sources, and is increased or decreased when the AAU is increased or decreased, respectively. Finally, the script quantified the fiber OI, which estimates fiber alignment with respect to the relative mean angle 
TABLE 1. Continued

\begin{tabular}{|c|c|c|c|c|c|c|}
\hline \multirow[b]{2}{*}{ Smoking $(\%)$} & \multicolumn{3}{|c|}{ Aortic insufficiency $(\%)$} & \multicolumn{3}{|c|}{ Aortic stenosis (\%) } \\
\hline & $1+$ & $\mathbf{2 - 3}+$ & $4+$ & Mild & Moderate & Severe \\
\hline $17 \%(1 / 6)$ & $50 \%(3 / 6)$ & $17 \%(1 / 6)$ & $0 \%(0 / 6)$ & $17 \%(1 / 6)$ & $0 \%(0 / 6)$ & $33 \%(2 / 6)$ \\
\hline $33 \%(2 / 6)$ & $0 \%(0 / 6)$ & $17 \%(1 / 6)$ & $17 \%(1 / 6)$ & $0 \%(0 / 6)$ & $33 \%(2 / 6)$ & $50 \%(3 / 6)$ \\
\hline
\end{tabular}

of fiber orientation in the matrix. OI $=1$ is indicative of a perfectly aligned fiber set in a parallel orientation or anisotropy, whereas $\mathrm{OI}=0.5$ indicates random fiber alignment or isotropy.

\section{Statistical Analysis}

Mann-Whitney $U$ pairwise tests $(P<.05)$ were performed using SigmaPlot software (SYSTAT Software, Chicago, Ill) to determine differences in collagen and elastin microarchitecture between the 2 aortic phenotypes (BAV-ATAA $\mathrm{n}=6$ and TAV-ATAA $\mathrm{n}=6$ ) in 18 locations of the aortic wall ( 3 RAD locations $\times 2$ planes $\times 3 \Theta$ regions). Data are presented as mean \pm standard error of the mean. Only significant differences $(P<.05)$ are presented.

\section{RESULTS}

\section{Assessment of Aneurysm Geometry}

Computed tomographic angiography revealed aneurysm geometries for all patients studied. ${ }^{27,28}$ Patients with type $1 \mathrm{R} / \mathrm{L}$ BAV morphotype $(\mathrm{n}=3)$ uniformly displayed a dilatation pattern limited to the tubular ascending aorta (type 2 aortic dilatation as previously described by others $^{27}$ ). Patients with the type 0 BAV morphotype $(\mathrm{n}=3)$ showed type 1 aortic dilatation involving the more proximal ascending aorta and aortic root. Conversely, all TAV patients $(\mathrm{n}=6)$ exhibited symmetrical dilatation involving the ascending aorta and proximal arch (ie, type 3 aortic dilatation). Most strikingly, the type $1 \mathrm{R} / \mathrm{L}$ BAV patients had an asymmetric dilatation pattern with exaggerated dilatation of the greater curve compared with all of the TAV patients or the type $0 \mathrm{BAV}$ patient. The notion that the BAV aortopathy, particularly type $1 \mathrm{R} / \mathrm{L} \mathrm{BAV}$, often manifests in an asymmetric pattern of dilatation has been previously raised by others. ${ }^{28,29}$ The patterns that were seen in the patients included in this study are shown in Figure 4.

\section{Elastin Fibers Were less Undulated for BAV-ATAA in Regions $\mathbf{N}$ and $\mathbf{R}$}

Image analysis of multiphoton micrographs corresponding to adv-med, media, and med-int layers revealed that medial elastin fibers were less oriented in the RAD direction in the region $\mathrm{N}$ of BAV-ATAA when compared with TAVATAA in the Z-RAD plane. This was demonstrated by a lower AAU for elastin fibers in the media layer of region $\mathrm{N}$ (Figure $5, A)\left(10.67^{\circ} \pm 1.35^{\circ}\right.$ vs $15.58^{\circ} \pm 1.91^{\circ}$; $P=.041$ ) (see also Table 2 for all the $P$ values derived from statistical analysis) and a lower AAU for elastin fibers in the med-int layer of region $\mathrm{R}$ (Figure $5, B)\left(9.83^{\circ} \pm 0.83^{\circ}\right.$ vs $\left.14.72^{\circ} \pm 1.64^{\circ} ; P=.015\right)$, in the Z-RAD and $\Theta$-RAD planes, respectively, in BAV-ATAA when compared with TAV-ATAA. Collagen fibers were found to be more oriented in the RAD direction in the outer layers of BAV-ATAA wall when compared with TAV-ATAA. This was demonstrated by a higher AAU for collagen fibers in the adv-med layer of region $\mathrm{N}$ (Figure 5, C) $\left(25.50^{\circ} \pm 7.18^{\circ}\right.$ vs $\left.13.50^{\circ} \pm 1.34^{\circ} ; P=.026\right)$, in the Z-RAD plane in BAV-ATAA when compared with TAV-ATAA.

\section{Collagen Fibers Were More Undulated for BAV-ATAA in Region $L$}

The same image analysis of multiphoton micrographs revealed that med-int collagen fibers were more oriented in the RAD direction in the region L of BAV-ATAA when compared with TAV-ATAA in the Z-RAD plane. This was demonstrated by a higher AAU for collagen fibers (Figure 6, A) $\left(18.67^{\circ} \pm 0.95^{\circ}\right.$ vs $14.56^{\circ} \pm 1.37^{\circ}$; $P=.041$ ), lower OI of collagen fibers (Figure 6, B) $\left(0.69^{\circ} \pm 0.02^{\circ}\right.$ vs $\left.0.77^{\circ} \pm 0.02^{\circ} ; P=.0026\right)$, higher percentage of RAD-oriented collagen fibers (Figure 6, C) $\left(2.82^{\circ} \pm 0.24^{\circ}\right.$ vs $\left.1.77^{\circ} \pm 0.25^{\circ} ; P=.041\right)$, and higher ratio of RAD over Z-oriented collagen fibers (Figure 6,D) $\left(0.29^{\circ} \pm 0.05^{\circ}\right.$ vs $\left.0.14^{\circ} \pm 0.03^{\circ} ; P=.026\right)$, in the Z-RAD plane of the med-int layer in BAV-ATAA when compared with TAV-ATAA.

We found differences in microarchitectural fiber orientation between BAV-ATAA and TAV-ATAA predominantly in the media and med-int layers between $\mathrm{L}$ and N-R regions, when screening all of the wall regions both across the thickness (med-int, media, and adv-med) and across the circumference ( $\mathrm{L}, \mathrm{N}$, and $\mathrm{R}$ ) in both Z-RAD and $\Theta$-RAD planes. Consistent with these analyses of the multiphoton imaging, histologic inspection of VerhoeffVan Gieson-stained specimens revealed a more disorganized elastin matrix in the $\mathrm{N}$ region when compared with the $\mathrm{L}$ region of the aortic media from $2 \mathrm{BAV}$ patients (Figure E5).

\section{DISCUSSION}

Our laboratory has generated a growing body of evidence that ATAAs arising in patients with BAV versus TAV involve distinct mechanisms of extracellular matrix remodeling indicative of differing vessel wall strength and 


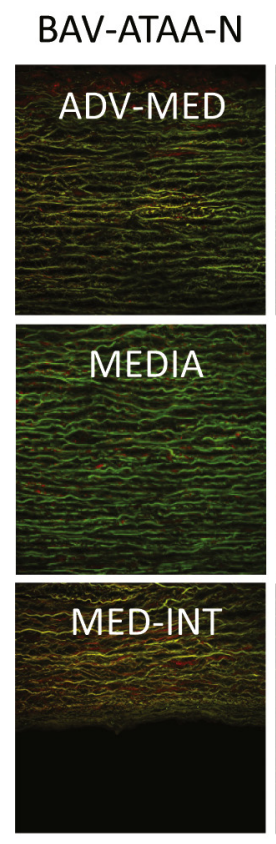

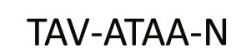
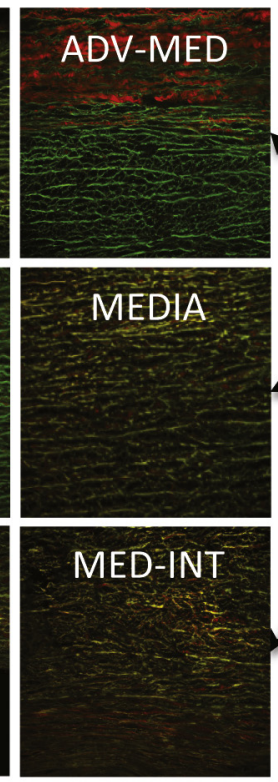
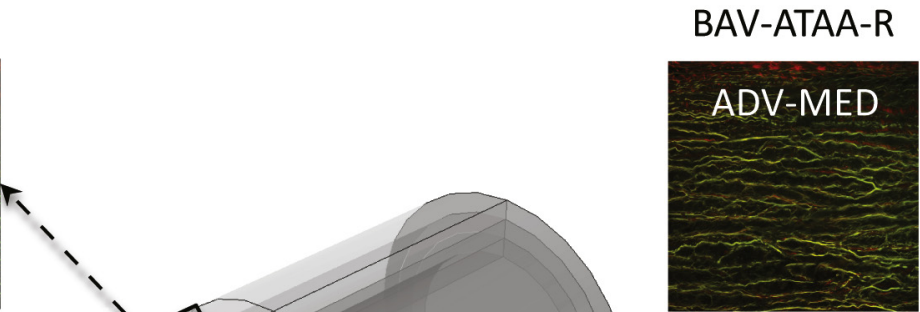

TAV-ATAA-R
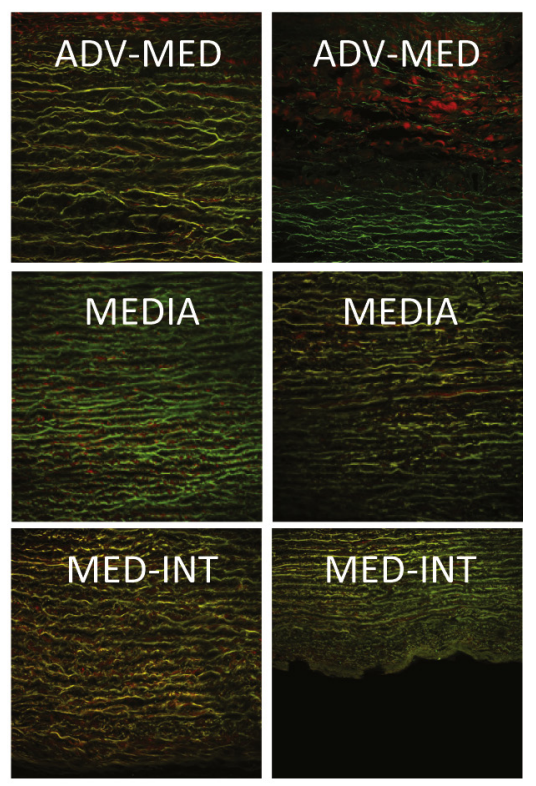

\section{RED: COLLAGEN GREEN: ELASTIN}

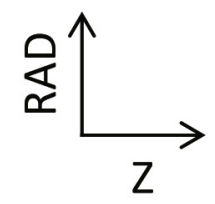

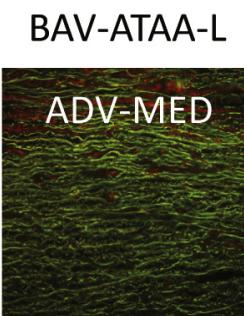
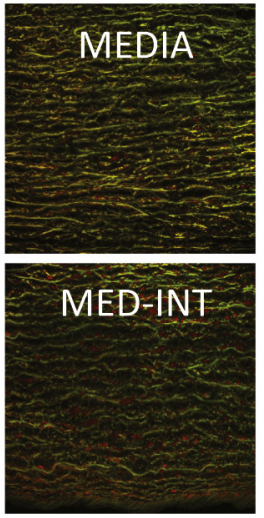

\section{TAV-ATAA-L}
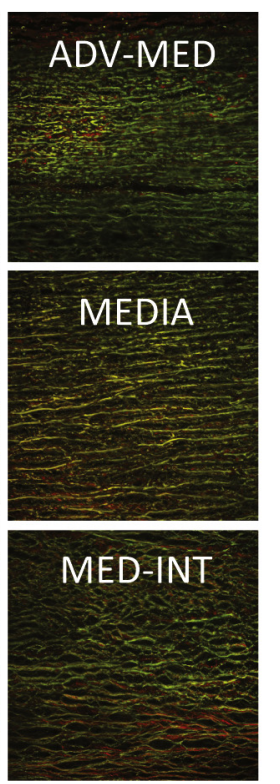

FIGURE 2. Example multiphoton microscopy images $\left(0.5 \times 0.5 \mathrm{~mm}^{2}\right.$ each) of collagen $($ red $)$ and elastin (green) fibers in the longitudinal $(Z)$ radial $(R A D)$ plane in 3 RAD-locations (adventitia-media [adv-med], media, and media-intima [med-int]) and in 3 circumferential $(\Theta)$ regions (left coronary sinus $[L]$, right coronary sinus $[R]$, and noncoronary sinus $[N]$ ) of both bicuspid valve ascending thoracic aortic aneurysm (BAV-ATAA) and tricuspid aortic valve ascending thoracic aortic aneurysm (TAV-ATAA). The 3 black-colored rectangles within the model tube indicate the imaging Z-RAD planes in the $3 \Theta-$ regions of the wall. $B A V-A T A A-N$, Bicuspid aortic valve ascending thoracic aortic aneurysm circumferential region with respect to noncoronary sinus; $T A V-A T A A-N$, tricuspid aortic valve ascending thoracic aortic aneurysm circumferential region with respect to noncoronary sinus; BAV-ATAA-R, bicuspid aortic valve ascending thoracic aortic aneurysm circumferential region with respect to right coronary sinus; TAV-ATAA-R, tricuspid aortic valve ascending thoracic aortic aneurysm circumferential region with respect to right coronary sinus; $B A V-A T A A-L$, bicuspid aortic valve ascending thoracic aortic aneurysm circumferential region with respect to left coronary sinus; TAV-ATAA- $L$, tricuspid aortic valve ascending thoracic aortic aneurysm circumferential region with respect to left coronary sinus.

dissection potential that are likely due to microarchitectural changes among collagen and elastin fibers. We recently reported a more aligned collagen and elastin fiber microarchitecture in the $\Theta-Z$ plane of BAV-ATAA specimens when compared with TAV-ATAA specimens, and these differences were associated with increased tensile strength $^{14}$ and altered collagen maturity in BAV-ATAA when compared with TAV-ATAA. ${ }^{21}$ We also previously characterized the aortic medial microarchitecture in the other 2 planes, Z-RAD and $\Theta-$ RAD, using multiphoton microscopy. ${ }^{22}$ Increased AAU of elastin fibers about the $\Theta$-axis and increased percentage of RAD-oriented elastin and 

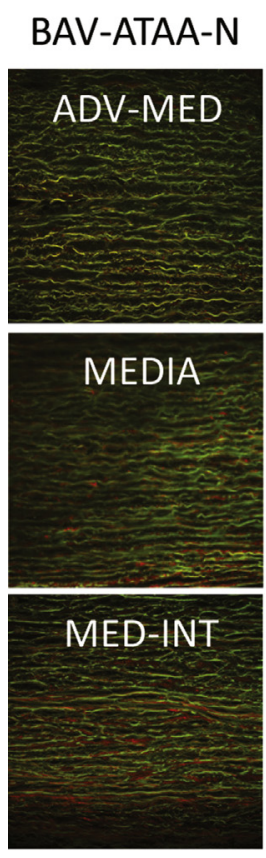

TAV-ATAA-N
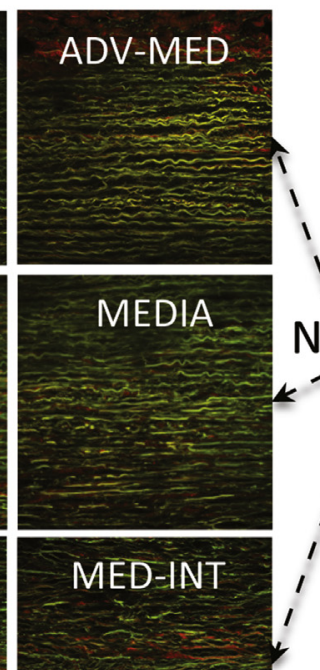
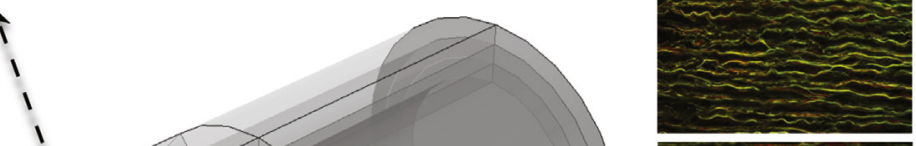
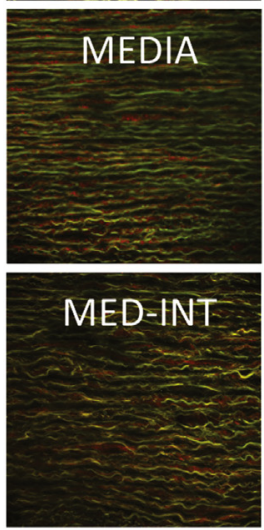

TAV-ATAA-R
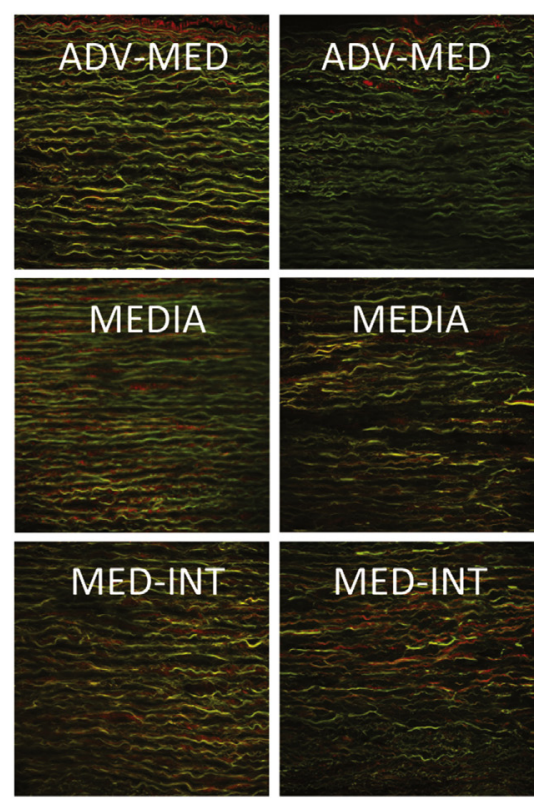

\section{RED: COLLAGEN}

GREEN: ELASTIN

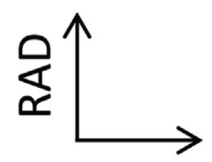

$\Theta$
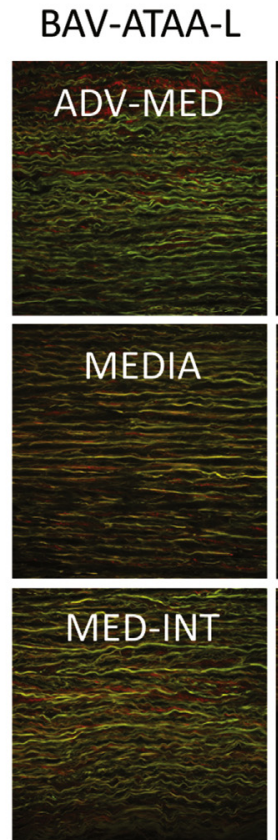

TAV-ATAA-L
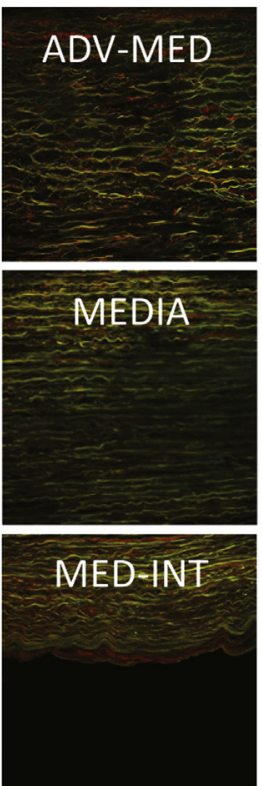

FIGURE 3. Example multiphoton microscopy images $\left(0.5 \times 0.5 \mathrm{~mm}^{2}\right.$ each) of collagen $($ red $)$ and elastin ( green $)$ fibers in the circumferential $(\Theta)$ radial $(R A D)$ plane in 3 RAD-locations (adventitia-media [adv-med], media, and media-intima [med-int]) and in $3 \Theta$-regions (left coronary sinus $[L]$, right coronary sinus $[R]$, and noncoronary sinus $[N])$ of both bicuspid valve ascending thoracic aortic aneurysm (BAV-ATAA) and tricupid aortic valve ascending thoracic aortic aneurysm (TAV-ATAA). The 3 black-colored rectangles within the model tube indicate the imaging $\Theta$-RAD planes in the $3 \Theta$-regions of the wall. BAV-ATAA-N, Bicuspid aortic valve ascending thoracic aortic aneurysm circumferential region with respect to noncoronary sinus; TAV-ATAA$N$, tricuspid aortic valve ascending thoracic aortic aneurysm circumferential region with respect to noncoronary sinus; $Z$, circumferential-longitudinal plane; $B A V-A T A A-R$, bicuspid aortic valve ascending thoracic aortic aneurysm circumferential region with respect to right coronary sinus; TAV-ATAA-R, tricuspid aortic valve ascending thoracic aortic aneurysm circumferential region with respect to right coronary sinus; $B A V-A T A A-L$, bicuspid aortic valve ascending thoracic aortic aneurysm circumferential region with respect to left coronary sinus; TAV-ATAA-L, tricuspid aortic valve ascending thoracic aortic aneurysm circumferential region with respect to left coronary sinus.

collagen fibers was noted in the outer media of BAV-ATAA when compared with a nonaneurysmal ascending aorta, which was not appreciated in TAV-ATAA. This finding is consistent with the increased tensile stretch noted at the inflection point of $\Theta$ strips of the outer delaminated half of
BAV-ATAA when compared with a nonaneurysmal ascending aorta, because more undulated fibers permit increased stretch before fibers become gradually engaged (ie, straightened). ${ }^{30}$ Furthermore, we found 2 discrepancies in the architecture of RAD-oriented fibers across the media 


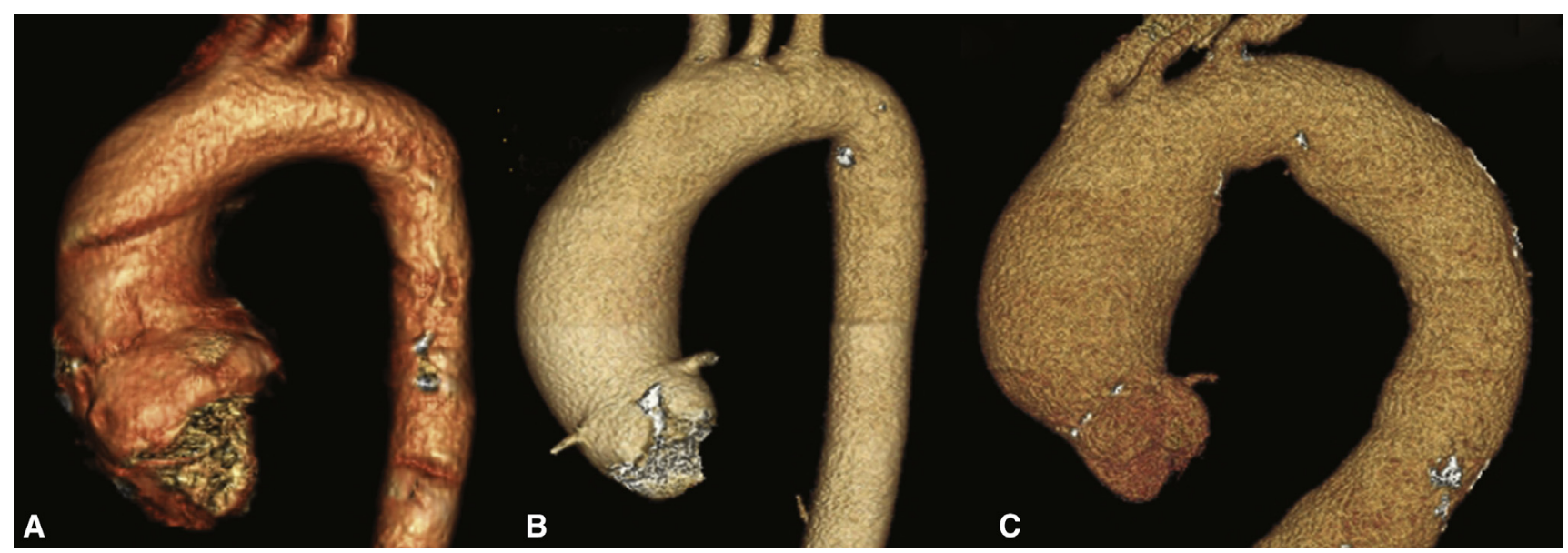

FIGURE 4. Three-dimensional renderings of preoperative computed tomographic angiograms from A, a patient with type 0 bicuspid aortic valve, B, a patient with type 1 bicuspid aortic valve with right/left morphotype, and $\mathrm{C}$, a patient with tricuspid aortic valve. The dilation patterns of all 3 patients with the type 1 right/left morphotype had the same pattern as depicted in $B$.

of TAV-ATAA with increasing age. Collagen AAU about the Z-axis was found to be increased within the outer-media. Secondly, collagen was found to be less RAD-oriented, and elastin was noted to be more oriented in the Z-axis within the inner media. Collectively, these findings provide evidence for different microarchitectural mechanisms of vascular remodeling that mediate vessel biomechanics in patients with BAV versus TAV.

Our current analysis, which was circumferential region-specific and thickness location-specific, revealed statistically significant differences in microarchitectural elastin fiber orientation between BAV-ATAA and TAVATAA in the media layer of region $\mathrm{N}$ and the med-int layer of region $\mathrm{R}$ of the Z-RAD plane. The finding of less RAD-oriented elastin fibers in the media layer of region $\mathrm{N}$ and the med-int layer of region R of BAV-ATAA may directly correlate with our earlier finding of decreased delamination strength (an ex vivo measure of aortic dissection potential) in BAV-ATAA compared with TAV-ATAA, ${ }^{15}$ where the artificial delamination had been performed across and parallel to the medial plane of the aortic wall $\mathrm{Z}$ and $\Theta$ strips. Nakashima and colleagues, ${ }^{31}$ who assessed the media of type A aortic dissections by histologic inspection, reported that medial microarchitecture was characterized by a decreased number of interlaminar elastin fibers. We show that the amount of RAD-oriented elastin fibers, which can be considered to be a subset of interlaminar fibers, was decreased in the medial layer of region $\mathrm{N}$ and the med-int layer of region $\mathrm{R}$ of BAV-ATAA compared with TAVATAA. Similarly, in a region-specific analysis by Sokolis and colleagues, ${ }^{32}$ inspection of the right-lateral region of TAV-ATAA (corresponding to region $\mathrm{N}$ in our study) revealed that elastin fibers of the media were noted to be aligned in the $\mathrm{Z}$-axis in the innermost media layer and primarily oriented in the $\Theta$ direction in the outermost media layer. These findings were distinctly unique compared with fiber orientation in both $\Theta$ and $\mathrm{Z}$ directions in the media of the other regions (ie, left-lateral, anterior, and posterior). This noted alteration in elastin orientation may be associated with microtears of elastin in the innermost media along the $\Theta$-axis. Our data demonstrate that the amount of RAD-oriented elastin fibers is decreased in the media layer of region $\mathrm{N}$ and the med-int layer of region $\mathrm{R}$ in the $\mathrm{Z}-\mathrm{RAD}$ tissue plane depending on bicuspid aortic valve morphology, and collectively these new findings may explain the asymmetric dilatation pattern commonly seen in patients with BAV-ATAA compared with those with TAV-ATAA.

We previously showed ${ }^{21}$ that the elastin content was similar among BAV-ATAAs and TAV-ATAAs, which was consistent with others' findings. ${ }^{16-19,31,33}$ We also discovered altered AAU for elastin, which implies altered percentage of RAD-oriented elastin in regions $\mathrm{N}$ and $\mathrm{R}$ among patients with BAV-ATAA and TAV-ATAA. The decreased AAU and subsequently decreased percentage of RAD-oriented elastin in regions $\mathrm{N}$ and $\mathrm{R}$ of BAV-ATAA could be due to remodeling of elastin fiber microarchitecture despite a total elastin content in ATAA tissue that is unchanged irrespective of age or aortic valve morphology ${ }^{4,34}$ and given the extremely low turnover rate of elastin in adults.

Considerations of collagen content in ascending aortopathies have led to conflicting reports. Prior studies have reported increased collagen content in thoracic aortic dissections, ${ }^{16,35}$ whereas others report no change in collagen content in BAV-ATAA. ${ }^{36}$ Della Corte and colleagues ${ }^{37}$ reported that collagen content is spatially dependent in BAV-ATAA. Despite increased levels of Type I collagen messenger RNA in BAV-ATAA, ${ }^{38}$ we also found that overall collagen content is comparable between 


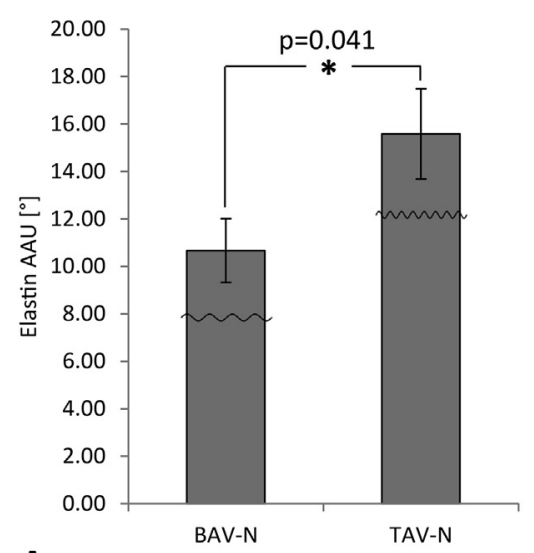

A

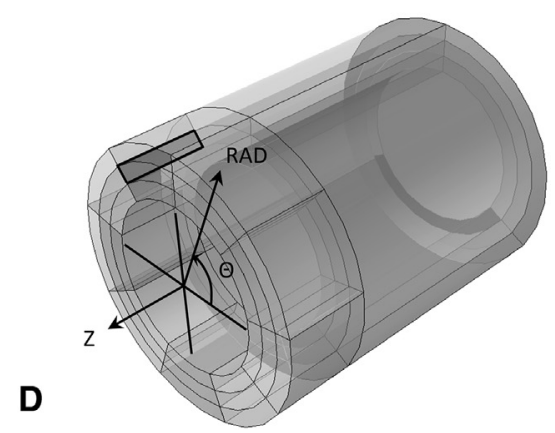

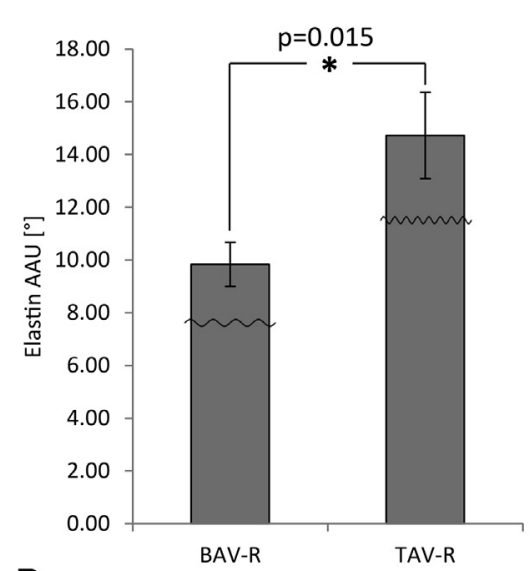

B

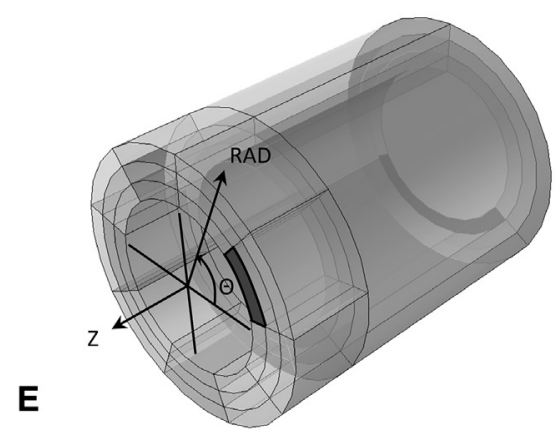

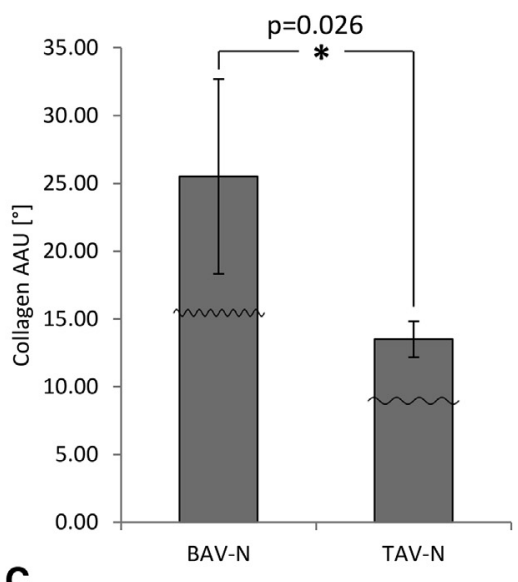

C

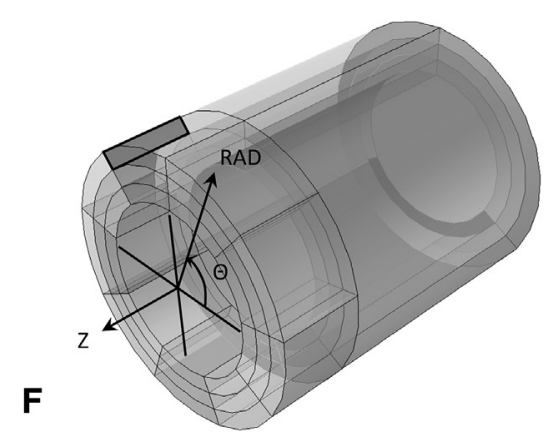

FIGURE 5. Results of Mann-Whitney $U$ tests (only the significant differences $[P<.05]$ are presented) for the comparison of amplitude of angular undulation between the 2 ascending thoracic aortic aneurysm phenotypes based on the output of the custom MATLAB written automated image-based analysis tool. Results are presented as mean \pm standard deviation. A, Amplitude of angular undulation of elastin in the media layer in the longitudinal $(Z)$ radial $(R A D)$ plane of region $\mathrm{N}, \mathrm{B}$, Amplitude of angular undulation of elastin in the media-intima (med-int) layer in the circumferential $(\Theta)$ RAD plane of region $\mathrm{R}$, and $\mathrm{C}$, Amplitude of angular undulation of collagen in the adventitia-media (adv-med) layer in the Z-RAD plane of region N. The little waveforms overlaid onto the bars indicate how the waviness of the fibers is affected. D, The black-colored rectangle within the model tube indicates the layer (media), region $(\mathrm{N})$, and plane (Z-RAD) across the thickness of the wall for the data of $A$. E, The black-colored rectangle within the model tube indicates the layer (med-int), region $(\mathrm{R})$, and plane $(\Theta-\mathrm{RAD})$ across the thickness of the wall for the data of $B$. F, The black-colored rectangle within the model tube indicates the layer (adv-med), region (N), and plane (Z-RAD) across the thickness of the wall for the data of $C$. *Significant difference. AAU, Amplitude of angular undulation; $B A V-N$, bicuspid aortic valve circumferential region with respect to noncoronary sinus; $T A V-N$, tricuspid aortic valve circumferential region with respect to noncoronary sinus; $B A V-R$, bicuspid aortic valve circumferential region with respect to right coronary sinus; $T A V-R$; tricuspid aortic valve circumferential region with respect to right coronary sinus.

TABLE 2. $P$ values of Mann-Whitney $U$ tests for amplitude of angular undulation (AAU) of collagen and elastin fibers between the 2 ascending thoracic aortic aneurysm (ATAA) phenotypes (bicuspid aortic valve [BAV] and tricuspid aortic valve [TAV]) in 18 locations of the aortic wall: 3 radial (RAD) locations (media-intima [med-int], media, and adventitia-media [adv-med]) $\times 2$ planes (Z-RAD and $\Theta$-RAD) $\times 3 \Theta$ regions (left coronary sinus $[\mathrm{L}]$, noncoronary sinus $[\mathrm{N}]$, and right coronary sinus $[\mathrm{R}])$. Statistical analysis showed that AAU is increased in region $\mathrm{L}$ and decreased in the media of region $\mathrm{N}$ and in the region $\mathrm{R}$ of BAV-ATAA compared with TAV-ATAA. The AAU is increased in the adv-med of region $\mathrm{N}$ of BAV-ATAA versus TAV-ATAA

\begin{tabular}{|c|c|c|c|c|c|c|}
\hline & Med-int & Med-int & Media & Media & Adv-med & Adv-med \\
\hline & Z-RAD & $\Theta-$ RAD & Z-RAD & $\Theta-$ RAD & Z-RAD & $\Theta$-RAD \\
\hline Collagen L & $.041 *$ & .310 & .132 & .937 & $.026^{*}$ & $.026^{*}$ \\
\hline Collagen $\mathrm{N}$ & .589 & .180 & .818 & .818 & $.026^{*}$ & .394 \\
\hline Collagen R & .132 & .937 & .240 & .589 & .818 & 699 \\
\hline Elastin L & .132 & .937 & .937 & .485 & .310 & .310 \\
\hline Elastin N & .485 & .589 & $.041 *$ & .240 & .818 & .485 \\
\hline Elastin R & .699 & $.015 *$ & 0.310 & .699 & .310 & .485 \\
\hline
\end{tabular}

Med-int, Media-intima layer; $A d v$-med, adventitia-media layer; $Z$ - $R A D$, longitudinal-radial plane; $\Theta$ - $R A D$, circumferential-radial plane; $L$, circumferential region with respect to left coronary sinus; $N$, circumferential region with respect to noncoronary sinus; $R$, circumferential region with respect to right coronary sinus. $*$ Significant difference. 

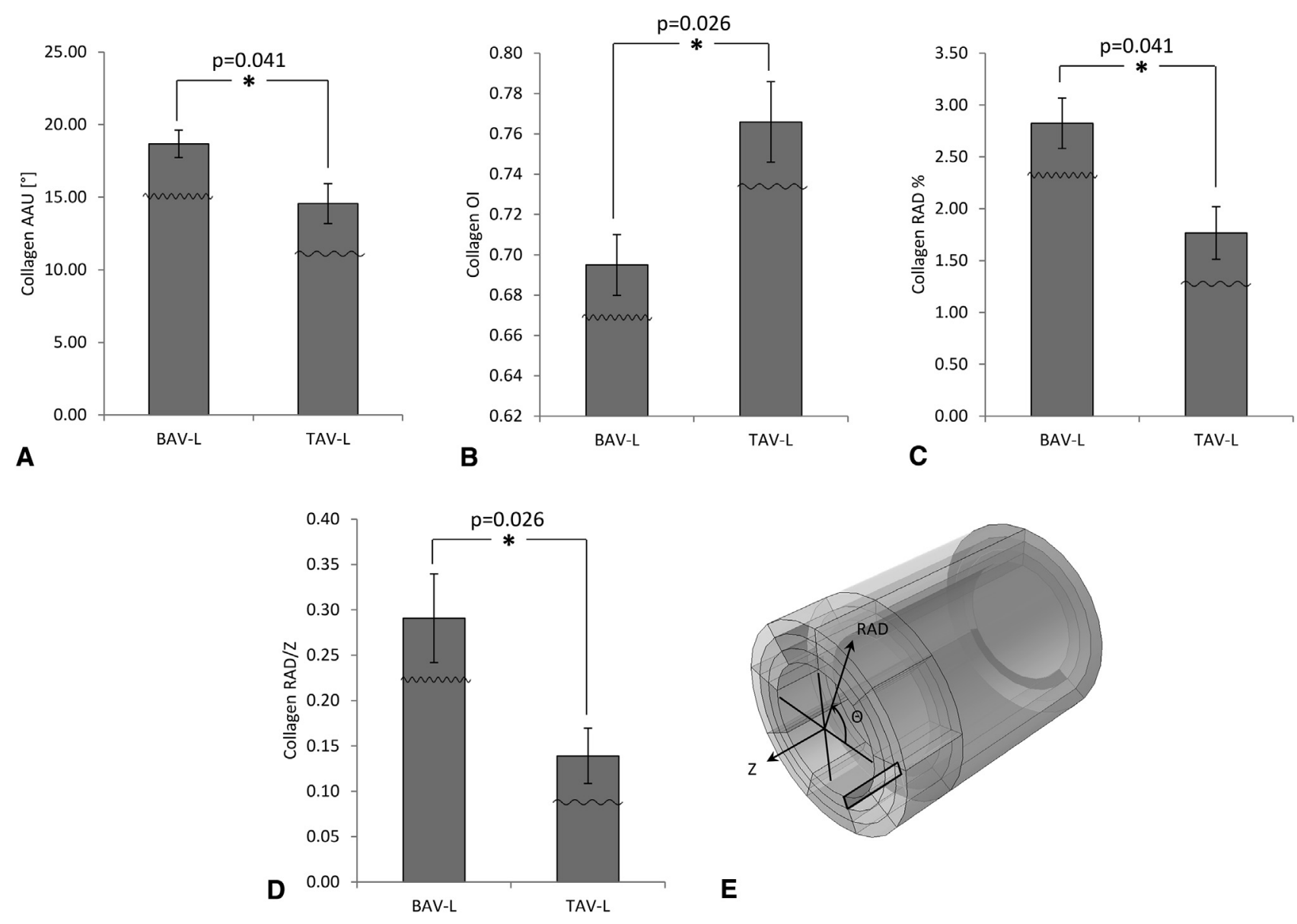

FIGURE 6. Results of Mann-Whitney $U$ tests (only the significant differences $[P<.05]$ are presented) for the comparison of collagen microarchitecture between the 2 ascending thoracic aortic aneurysm phenotypes in the media-intima (med-int) layer in the longitudinal $(Z) \operatorname{radial}(R A D)$ plane of region $L$ based on the output of the custom MATLAB written automated image-based analysis tool. A Amplitude of angular undulation of collagen (same trend also observed for collagen amplitude of angular undulation between bicuspid aortic valve $(B A V)$ and tricuspid aortic valve (TAV) in the adventitia-media $(a d v-m e d)$ layer of Z-RAD and circumferential $(\Theta)$ RAD planes of region L; see first row of Table 2 for $P=.026$ in both cases; bar graphs not shown here), $B$ Orientation index $(O I)$ of collagen fibers, $C$, percent of collagen fibers oriented in the RAD-direction (same trend also observed for percent of elastin fibers oriented in the RAD-direction between BAV and TAV $[P=.041]$ in the same layer [med-int], plane [Z-RAD], and region [L]; bar graphs not shown here), and $D$ ratio of collagen fibers oriented in the RAD over Z-direction. The little waveforms overlaid onto the bars indicate how the waviness of the fibers is affected. $E$, The black-colored rectangle within the model tube indicates the layer (med-int), region (L), and plane (Z-RAD) across the thickness of the wall. Results are presented as mean \pm standard deviation. * Significant difference. $A A U$, Amplitude of angular undulation; $O I$, orientation index; $B A V-L$, bicuspid aortic valve circumferential region with respect to left coronary sinus; $T A V-L$, tricuspid aortic valve circumferential region with respect to left coronary sinus.

BAV-ATAA and TAV-ATAA. ${ }^{14,21}$ Despite the varied assessments of total collagen content in ATAAs, aging also appears to play a role in collagen architecture. . $^{44,39}$ In the current study, we found that aortic valve phenotype influenced the collagen microarchitecture in $2 \Theta$ regions of the ATAA wall: L and N. It was found that collagen AAU was increased in the med-int layer (as well as the adv-med layer; data not shown) of region $\mathrm{L}$ and the advmed layer of region N. Similar to the findings noted with elastin, the observed increased AAU and subsequently increased percentage of RAD-oriented collagen fibers in region $\mathrm{L}$ and the adv-med layer of region $\mathrm{N}$ could be achieved by remodeling of collagen fiber microarchitecture rather than an increase in total collagen content.

\section{Limitations}

We recognize that this study is limited by its small sample size (12 patients) despite rigorous multiphoton microscopic analysis (18 aortic sections screened, imaged, and analyzed per patient sample). Although we would have liked to have included aortic wall samples from patients without a dilatated BAV undergoing aortic valve replacement, collection of ascending aortic specimens from all $3 \Theta$ regions needed for this regionally based analysis was not feasible in patients for whom aortic replacement was not planned. Despite the lack of available aortic tissue in this cohort, we believe that microarchitectural changes occur early in the progression of the BAV aortopathy because our inspection of nondilatated specimens in a region-nonspecific 
manner (ie, small samples taken along the aortotomy site during aortic valve replacement) revealed findings of remodeling similar to that seen in the BAV-ATAA samples included in our present study. ${ }^{21}$ Future work will include interrogations of the other, less-prevalent BAV morphotypes (eg, type $1 \mathrm{R} / \mathrm{N}$ and type $1 \mathrm{~L} / \mathrm{N}$ ) and will explore the potential association of raphe position on region-specific microarchitectural changes. Correlating the elastin percentage of RAD to a measure of asymmetric aortic dilatation among all BAV morphotypes (compared with TAV controls) would strengthen the effect of our current findings.

\section{CONCLUSIONS}

Differences in fiber microarchitecture between BAV-ATAA and TAV-ATAA are evident between regions N-R and L. Elastin fiber microarchitecture can be characterized as less RAD-oriented in regions N-R, whereas collagen fiber microarchitecture can be characterized as more RAD-oriented in region L, in BAV-ATAA when compared with TAV-ATAA. The region-specific architectural profile in BAV-ATAA may be driven by distinct mechanisms of vascular remodeling that are a consequence of valve morphotype-specific hemodynamic stresses/forces (eg, blood flow-induced shear stress acting on the luminal surface) and contribute to an asymmetric dilatation pattern and/or convey a different regional propensity for delamination. These findings offer an explanation for the observed tendency of type $1 \mathrm{R} / \mathrm{L}$ BAV-ATAA to dilate asymmetrically toward the greater curvature of the aorta (regions $\mathrm{N}$ and $\mathrm{R}$ ) compared with TAV-ATAA and encourage more rigorous interrogation of the microarchitectural changes within BAV-specific aneurysms. When combined with additional region-specific (across the wall circumference and thickness) delamination or tensile biomechanical testing, this work improves our understanding of the biomechanical mechanisms of aortic aneurysm and dissection potential. Collectively, our hope is that a patient-specific simulation tool can be developed for enabling the prediction of aortic remodeling and disease progression. Such a tool could improve risk assessment for aortic catastrophe and direct therapy for patients with ATAA.

\section{Conflict of Interest Statement}

Authors have nothing to disclose with regard to commercial support.

The authors thank Michael Eskay, Benjamin Green, and Jennifer Hill for providing assistance in processing human ATA tissue. The authors also thank Dr. Andrew Althouse for assisting with statistical analysis of the data.

\section{References}

1. Bilen E, Akcay M, Bayram NA, Kocak U, Kurt M, Tanboga IH, et al. Aortic elastic properties and left ventricular diastolic function in patients with isolated bicuspid aortic valve. J Heart Valve Dis. 2012;21:189-94.

2. Ikonomidis JS, Ruddy JM, Benton SM Jr, Arroyo J, Brinsa TA, Stroud RE, et al. Aortic dilatation with bicuspid aortic valves: cusp fusion correlates to matrix metalloproteinases and inhibitors. Ann Thorac Surg. 2012;93:457-63.
3. Plaisance BR, Winkler MA, Attili AK, Sorrell VL. Congenital bicuspid aortic valve first presenting as an aortic aneurysm. Am J Med. 2012;125:e5-7.

4. Gleason TG. Heritable disorders predisposing to aortic dissection. Semin Thorac Cardiovasc Surg. 2005;17:274-81.

5. Ward C. Clinical significance of the bicuspid aortic valve. Heart. 2000;83:81-5.

6. Keane MG, Wiegers SE, Plappert T, Pochettino A, Bavaria JE, Sutton MG. Bicuspid aortic valves are associated with aortic dilatation out of proportion to coexistent valvular lesions. Circulation. 2000;102(19 Suppl 3):III35-9.

7. Davies RR, Goldstein LJ, Coady MA, Tittle SL, Rizzo JA, Kopf GS, et al. Yearly rupture or dissection rates for thoracic aortic aneurysms: Simple prediction based on size. Ann Thorac Surg. 2002;73:17-27.

8. Svensson LG, Adams DH, Bonow RO, Kouchoukos NT, Miller DC, O’Gara PT, et al. Aortic valve and ascending aorta guidelines for management and quality measures: executive summary. Ann Thorac Surg. 2013;95:1491-505.

9. Nishimura RA, Otto CM, Bonow RO, Carabello BA, Erwin JP III, Guyton RA, et al. 2014 AHA/ACC guideline for the management of patients with valvular heart disease: a report of the American College of Cardiology/American Heart Association Task Force on practice guidelines. Circulation. 2014;129:e521-643.

10. den Reijer PM, Sallee D III, van der Velden P, Zaaijer ER, Parks WJ, Ramamurthy S, et al. Hemodynamic predictors of aortic dilatation in bicuspid aortic valve by velocity-encoded cardiovascular magnetic resonance. J Cardiovasc Magn Res. 2010;12:4.

11. Hope MD, Hope TA, Crook SE, Ordovas KG, Urbania TH, Alley MT, et al. 4D flow CMR in assessment of valve-related ascending aortic disease. JACC Cardiovasc Imag. 2011;4:781-7.

12. Hope MD, Hope TA, Meadows AK, Ordovas KG, Urbania TH, Alley MT, et al. Bicuspid aortic valve: four-dimensional MR evaluation of ascending aortic systolic flow patterns. Radiology. 2010;255:53-61.

13. Weigang E, Kari FA, Beyersdorf F, Luehr M, Etz CD, Frydrychowicz A, et al. Flow-sensitive four-dimensional magnetic resonance imaging: flow patterns in ascending aortic aneurysms. Eur J Cardiothorac Surg. 2008;34:11-6.

14. Pichamuthu JE, Phillippi JA, Cleary DA, Chew DW, Hempel J, Vorp DA, et al. Differential tensile strength and collagen composition in ascending aortic aneurysms by aortic valve phenotype. Ann Thorac Surg. 2013;96:2147-54.

15. Pasta S, Phillippi JA, Gleason TG, Vorp DA. Effect of aneurysm on the mechanical dissection properties of the human ascending thoracic aorta. J Thorac Cardiovasc Surg. 2012;143:460-7.

16. de Figueiredo Borges L, Jaldin RG, Dias RR, Stolf NA, Michel JB, Gutierrez PS. Collagen is reduced and disrupted in human aneurysms and dissections of ascending aorta. Hum Pathol. 2008;39:437-43.

17. Nakashima Y, Kurozumi T, Sueishi K, Tanaka K. Dissecting aneurysm: a clinicopathologic and histopathologic study of 111 autopsied cases. Hum Pathol. 1990;21:291-6.

18. Wang X, LeMaire SA, Chen L, Carter SA, Shen YH, Gan Y, et al. Decreased expression of fibulin-5 correlates with reduced elastin in thoracic aortic dissection. Surgery. 2005;138:352-9.

19. Sariola H, Viljanen T, Luosto R. Histological pattern and changes in extracellular matrix in aortic dissections. J Clin Pathol. 1986;39:1074-81.

20. Saruk M, Eisenstein R. Aortic lesion in Marfan syndrome: the ultrastructure of cystic medial degeneration. Arch Pathol Lab Med. 1977;101:74-7.

21. Phillippi JA, Green BR, Eskay MA, Kotlarczyk MP, Hill MR, Robertson AM, et al. Mechanism of aortic medial matrix remodeling is distinct in patients with bicuspid aortic valve. J Thorac Cardiovasc Surg. 2014;147:1056-64.

22. Tsamis A, Phillippi JA, Koch RG, Pasta S, D'Amore A, Watkins SC, et al. Fiber micro-architecture in the longitudinal-radial and circumferential-radial planes of ascending thoracic aortic aneurysm media. J Biomech. 2013;46:2787-94.

23. Jiang X, Zhong J, Liu Y, Yu H, Zhuo S, Chen J. Two-photon fluorescence and second-harmonic generation imaging of collagen in human tissue based on multiphoton microscopy. Scanning. 2011;33:53-6.

24. Konig K, Schenke-Layland K, Riemann I, Stock UA. Multiphoton autofluorescence imaging of intratissue elastic fibers. Biomaterials. 2005;26:495-500.

25. D'Amore A, Stella JA, Wagner WR, Sacks MS. Characterization of the complete fiber network topology of planar fibrous tissues and scaffolds. Biomaterials. 2010;31:5345-54.

26. Karlon WJ, Covell JW, McCulloch AD, Hunter JJ, Omens JH. Automated measurement of myofiber disarray in transgenic mice with ventricular expression of ras. Anatomic Record. 1998;252:612-25.

27. Kang JW, Song HG, Yang DH, Baek S, Kim DH, Song JM, et al. Association between bicuspid aortic valve phenotype and patterns of valvular dysfunction and bicuspid aortopathy: comprehensive evaluation using MDCT and echocardiography. JACC Cardiovasc Imaging. 2013;6:150-61. 
28. Cotrufo M, Della Corte A. The association of bicuspid aortic valve disease with asymmetric dilatation of the tubular ascending aorta: identification of a definite syndrome. J Cardiovasc Med (Hagerstown). 2009;10:291-7.

29. Cotrufo M, Della Corte A, De Santo LS, Quarto C, De Feo M, Romano G, et al. Different patterns of extracellular matrix protein expression in the convexity and the concavity of the dilated aorta with bicuspid aortic valve: preliminary results. $J$ Thorac Cardiovase Surg. 2005;130:504-11.

30. Tsamis A, Rachev A, Stergiopulos N. A constituent-based model of age-related changes in conduit arteries. Am J Physiol Heart C. 2011;301:H1286-301.

31. Nakashima Y, Shiokawa Y, Sueishi K. Alterations of elastic architecture in human aortic dissecting aneurysm. Lab Invest. 1990;62:751-60.

32. Sokolis DP, Kritharis EP, Giagini AT, Lampropoulos KM, Papadodima SA, Iliopoulos DC. Biomechanical response of ascending thoracic aortic aneurysms: association with structural remodelling. Comput Methods Biomech Biomed Engin. 2012;15:231-48.

33. Cattell MA, Hasleton PS, Anderson JC. Increased elastin content and decreased elastin concentration may be predisposing factors in dissecting aneurysms of human thoracic aorta. Cardiovasc Res. 1993;27:176-81.

34. Tadros TM, Klein MD, Shapira OM. Ascending aortic dilatation associated with bicuspid aortic valve pathophysiology, molecular biology, and clinical implications. Circulation. 2009;119:880-90.
35. Wang X, LeMaire SA, Chen L, Shen YH, Gan Y, Bartsch H, et al. Increased collagen deposition and elevated expression of connective tissue growth factor in human thoracic aortic dissection. Circulation. 2006;114(1 Suppl):I200-5.

36. Fedak PW, de Sa MP, Verma S, Nili N, Kazemian P, Butany J, et al. Vascular matrix remodeling in patients with bicuspid aortic valve malformations: implications for aortic dilatation. J Thorac Cardiovasc Surg. 2003;126: 797-806.

37. Della Corte A, Quarto C, Bancone C, Castaldo C, Di Meglio F, Nurzynska D, et al. Spatiotemporal patterns of smooth muscle cell changes in ascending aortic dilatation with bicuspid and tricuspid aortic valve stenosis: focus on cell-matrix signaling. J Thorac Cardiovasc Surg. 2008;135:8-18. e1-2.

38. Phillippi JA, Eskay MA, Kubala AA, Pitt BR, Gleason TG. Altered oxidative stress responses and increased type I collagen expression in bicuspid aortic valve patients. Ann Thorac Surg. 2010;90:1893-8.

39. Tsamis A, Krawiec JT, Vorp DA. Elastin and collagen fibre microstructure of the human aorta in ageing and disease: a review. J R Soc Interface. 2013;10:20121004.

Key Words: bicuspid aortic valve, aortopathy, aortic wall microarchitecture, ascending aortic aneurysm, collagen, elastin

\title{
EDITORIAL COMMENTARY
}

\section{Real estate of the bicuspid aorta: Location, location, location!}

\author{
Paul W. M. Fedak, MD, PhD, ${ }^{\mathrm{a}, \mathrm{b}}$ and David G. Guzzardi, BSc ${ }^{\mathrm{a}}$
}

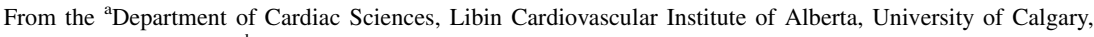
Calgary, Canada; and ${ }^{\mathrm{b}}$ Bluhm Cardiovascular Institute, Northwestern University, Chicago, Ill.

Disclosures: Authors have nothing to disclose with regard to commercial support.

Received for publication Feb 20, 2016; accepted for publication Feb 23, 2016; available ahead of print March 23, 2016.

Address for reprints: Paul W. M. Fedak, MD, PhD, Room 880, 1403-29 St NW, Calgary, Alberta, Canada T2N 2T9 (E-mail: paul.fedak@gmail.com).

J Thorac Cardiovasc Surg 2016;151:1728-9

$0022-5223 / \$ 36.00$

Copyright (c) 2016 by The American Association for Thoracic Surgery

http://dx.doi.org/10.1016/j.jtcvs.2016.02.048
}

\section{E}

As the most common congenital heart defect, bicuspid aortic valve (BAV) disease is a significant health care burden. ${ }^{1}$ With an understanding that patients with BAVs may have a predisposition to aortic complications secondary to an underlying aortopathy, surgeons often are challenged with decisions to prophylactically resect a moderately dilated aorta in those with BAVs. Surgeons' perspectives on the cause and mechanisms of bicuspid aortopathy may influence the timing and extent of their surgical approach. ${ }^{2,3}$ Bicuspid aortopathy is characterized by extracellular matrix (ECM) remodeling resulting in degeneration of elastic and collagen fibers within the aortic wall. ${ }^{4}$ Does the remodeling process of bicuspid

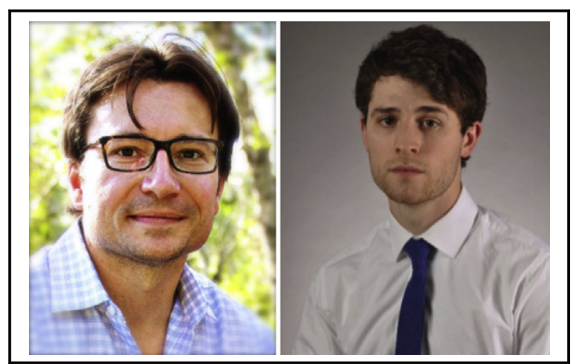

Paul W. M. Fedak, MD, PhD, and David G. Guzzardi, BSc

\section{Central Message}

Remodeling of ECM in the aortas of patients with BAVs is distinct from patients with TAVs.

See Article page 1718.

aortopathy reflect a distinct disease entity compared with patients with tricuspid aortic valves (TAVs)? Should these patients be resected more aggressively? Some recent data challenge this long-held belief and suggest that histologic changes are not more severe in the BAV compared with the TAV. ${ }^{5}$ Studies that help define the molecular, cellular, and structural alterations within the aorta of patients with 

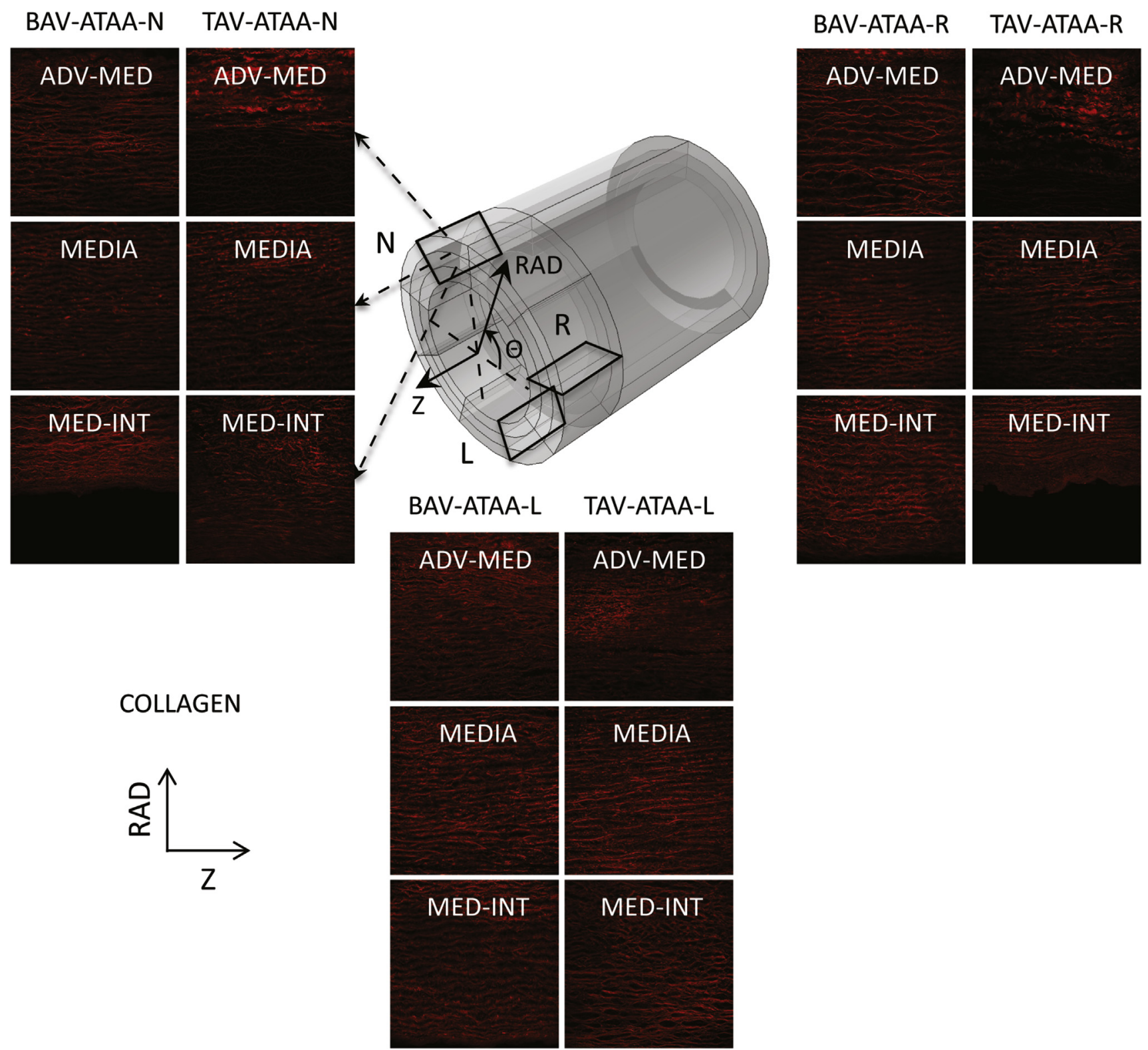

FIGURE E1. The collagen (red) channel of Figure 2. BAV-ATAA-N, Bicuspid aortic valve ascending thoracic aortic aneurysm circumferential region with respect to noncoronary sinus; $T A V-A T A A-N$, tricuspid aortic valve ascending thoracic aortic aneurysm circumferential region with respect to noncoronary sinus; $A d v$-med, adventitia-media layer; Med-int, media-intima layer; $N$, circumferential region with respect to noncoronary sinus $R A D$, radial; $Z$, circumferential-longitudinal plane; $\Theta$, circumferential; $R$, circumferential region with respect to right coronary sinus $L$, circumferential region with respect to left coronary sinus; $B A V$ - $A T A A-R$, bicuspid aortic valve ascending thoracic aortic aneurysm circumferential region with respect to right coronary sinus; $T A V-A T A A-R$, tricuspid aortic valve ascending thoracic aortic aneurysm circumferential region with respect to right coronary sinus; $B A V-A T A A-L$, bicuspid aortic valve ascending thoracic aortic aneurysm circumferential region with respect to left coronary sinus; TAV-ATAA-L, tricuspid aortic valve ascending thoracic aortic aneurysm circumferential region with respect to left coronary sinus. 


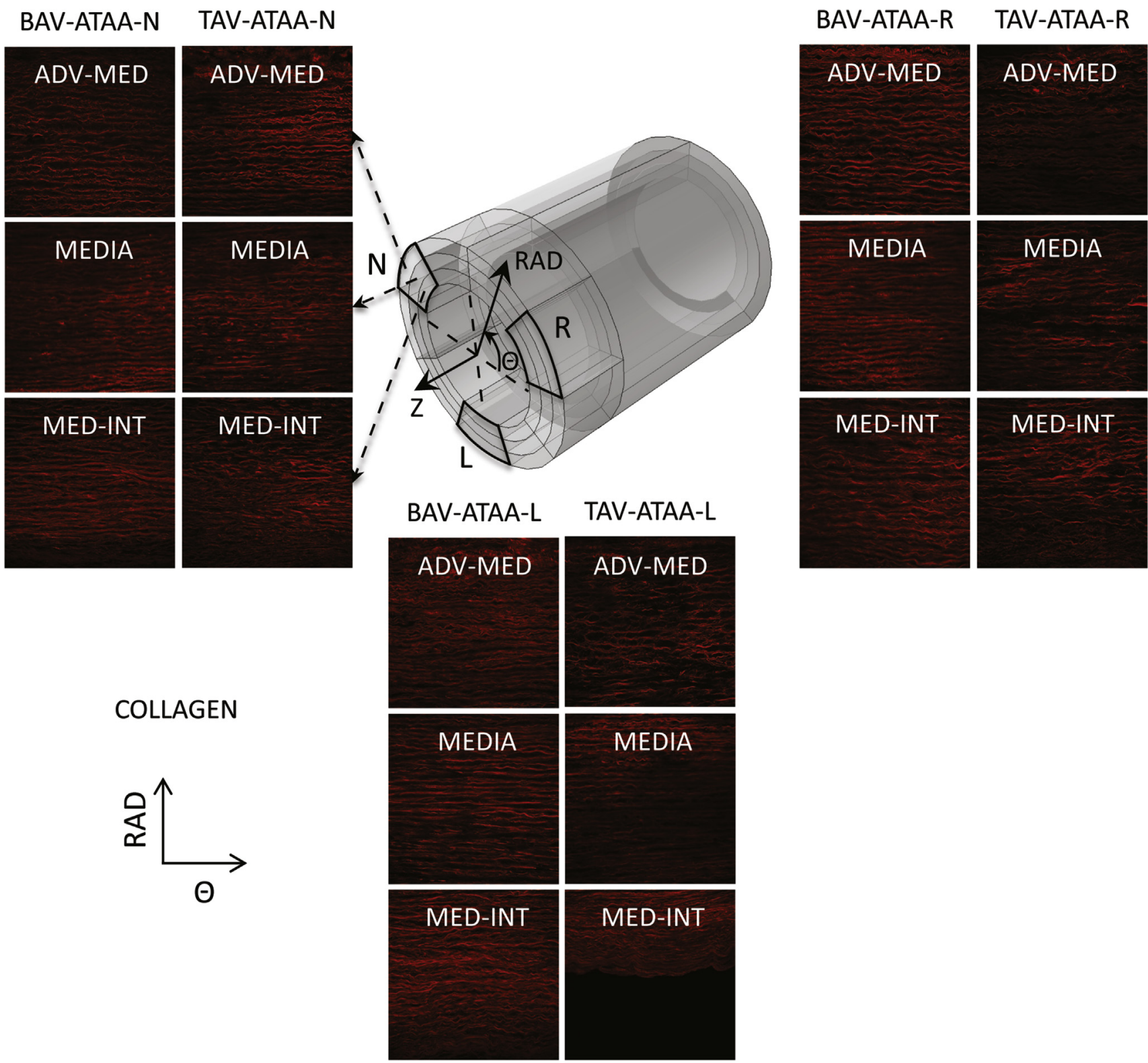

FIGURE E2. The collagen (red) channel of Figure 3. BAV-ATAA-N, Bicuspid aortic valve ascending thoracic aortic aneurysm circumferential region with respect to noncoronary sinus; $T A V-A T A A-N$, tricuspid aortic valve ascending thoracic aortic aneurysm circumferential region with respect to noncoronary sinus; $A d v$-med, adventitia-media layer; Med-int, media-intima layer; $N$, circumferential region with respect to noncoronary sinus $R A D$, radial; $Z$, circumferential-longitudinal plane; $\Theta$, circumferential; $R$, circumferential region with respect to right coronary sinus $L$, circumferential region with respect to left coronary sinus; $B A V-A T A A-R$, bicuspid aortic valve ascending thoracic aortic aneurysm circumferential region with respect to right coronary sinus; $T A V-A T A A-R$, tricuspid aortic valve ascending thoracic aortic aneurysm circumferential region with respect to right coronary sinus; $B A V-A T A A-L$, bicuspid aortic valve ascending thoracic aortic aneurysm circumferential region with respect to left coronary sinus; TAV-ATAA-L, tricuspid aortic valve ascending thoracic aortic aneurysm circumferential region with respect to left coronary sinus. 


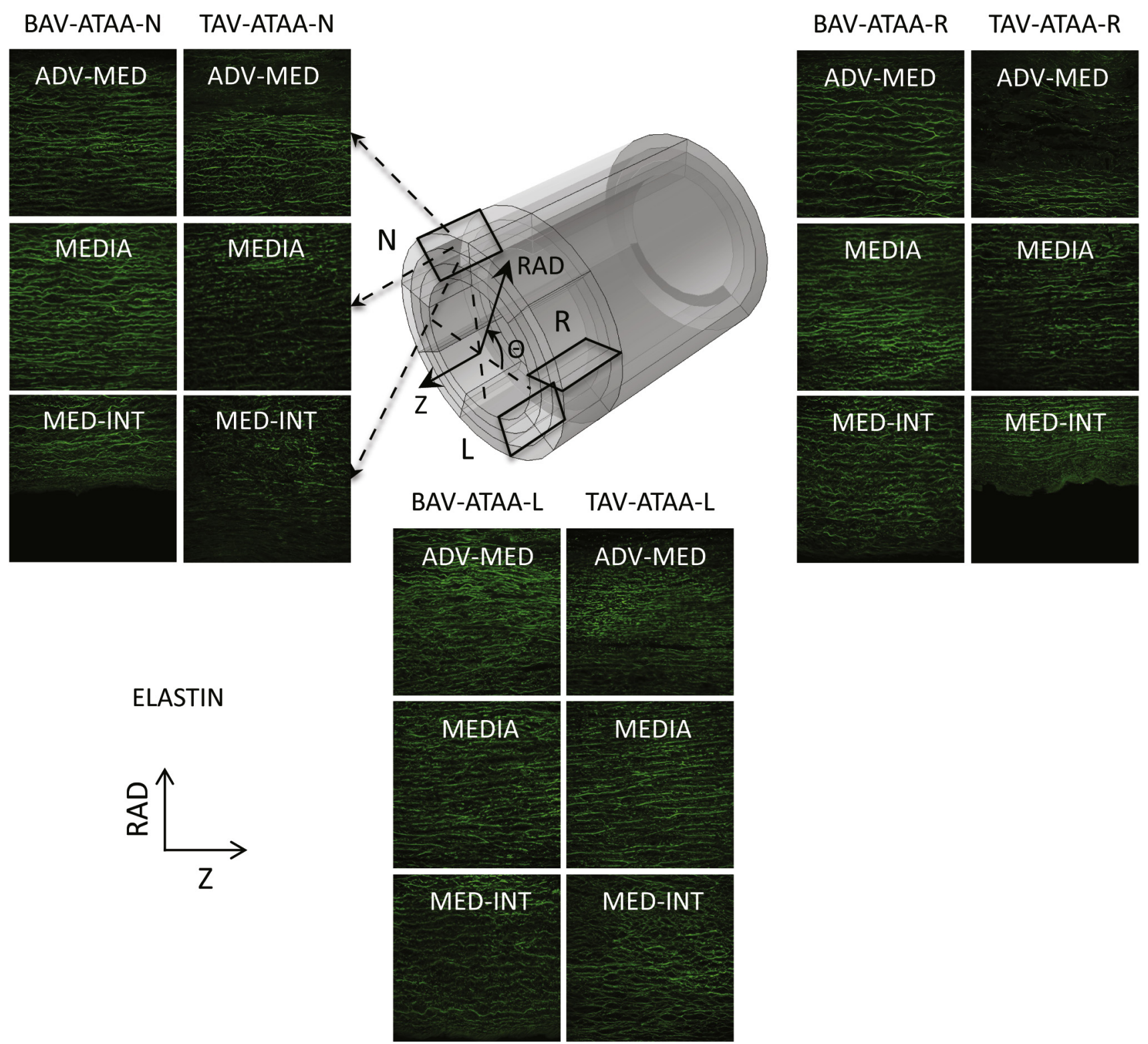

FIGURE E3. The elastin (green) channel of Figure 2. BAV-ATAA-N, Bicuspid aortic valve ascending thoracic aortic aneurysm circumferential region with respect to noncoronary sinus; $T A V-A T A A-N$, tricuspid aortic valve ascending thoracic aortic aneurysm circumferential region with respect to noncoronary sinus; $A d v$-med, adventitia-media layer; Med-int, media-intima layer; $N$, circumferential region with respect to noncoronary sinus $R A D$, radial; $Z$, circumferential-longitudinal plane; $\Theta$, circumferential; $R$, circumferential region with respect to right coronary sinus $L$, circumferential region with respect to left coronary sinus; $B A V-A T A A-R$, bicuspid aortic valve ascending thoracic aortic aneurysm circumferential region with respect to right coronary sinus; $T A V-A T A A-R$, tricuspid aortic valve ascending thoracic aortic aneurysm circumferential region with respect to right coronary sinus; BAV-ATAA-L, bicuspid aortic valve ascending thoracic aortic aneurysm circumferential region with respect to left coronary sinus; TAV-ATAA-L, tricuspid aortic valve ascending thoracic aortic aneurysm circumferential region with respect to left coronary sinus. 

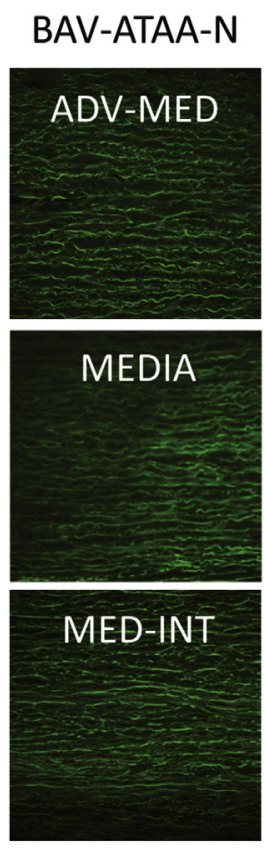
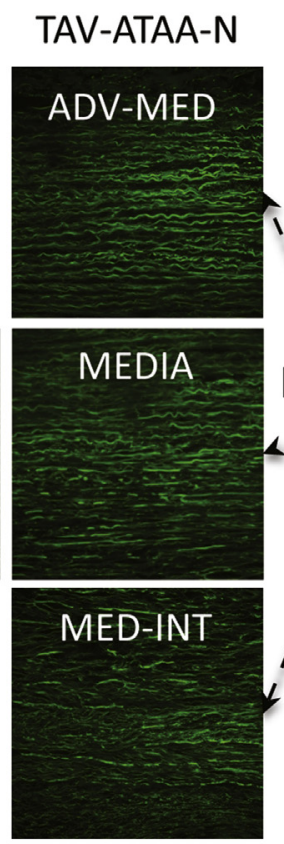
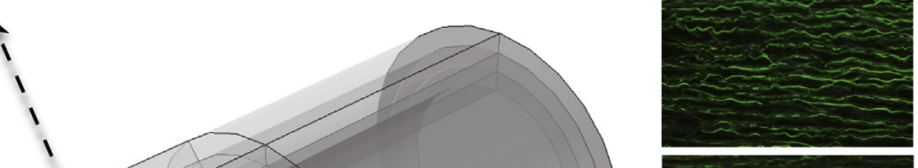
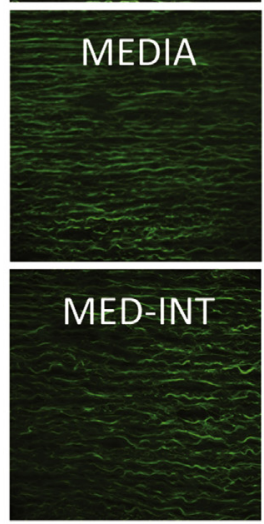

TAV-ATAA-R
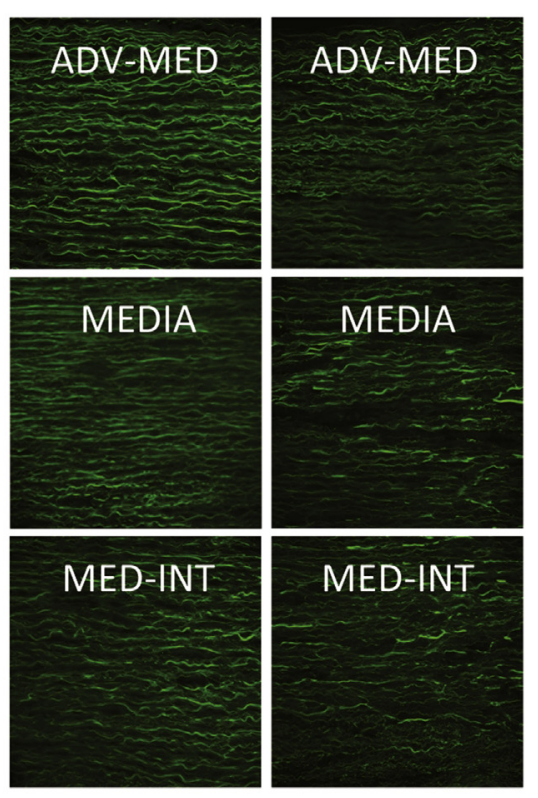

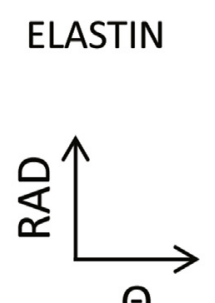

$\Theta$
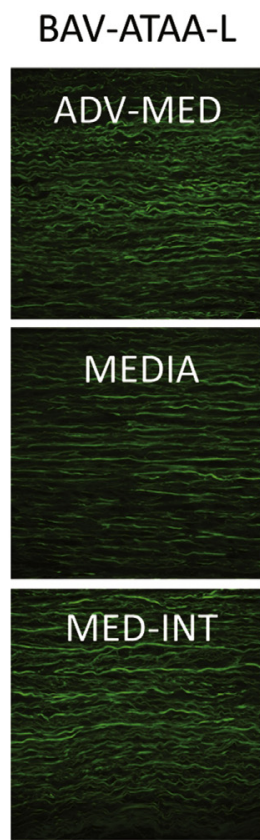

TAV-ATAA-L

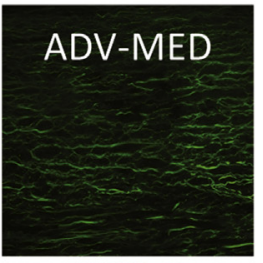

MEDIA

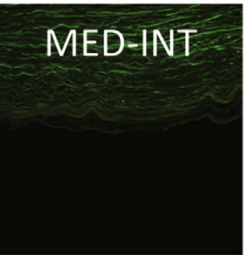

FIGURE E4. The elastin (green) channel of Figure 3. BAV-ATAA- $N$, Bicuspid aortic valve ascending thoracic aortic aneurysm circumferential region with respect to noncoronary sinus; $T A V-A T A A-N$, tricuspid aortic valve ascending thoracic aortic aneurysm circumferential region with respect to noncoronary sinus; $A d v$-med, adventitia-media layer; Med-int, media-intima layer; $N$, circumferential region with respect to noncoronary sinus $R A D$, radial; $Z$, circumferential-longitudinal plane; $\Theta$, circumferential; $R$, circumferential region with respect to right coronary sinus $L$, circumferential region with respect to left coronary sinus; $B A V$ - $A T A A-R$, bicuspid aortic valve ascending thoracic aortic aneurysm circumferential region with respect to right coronary sinus; $T A V$ - $A T A A-R$, tricuspid aortic valve ascending thoracic aortic aneurysm circumferential region with respect to right coronary sinus; $B A V$ - $A T A A-L$, bicuspid aortic valve ascending thoracic aortic aneurysm circumferential region with respect to left coronary sinus; $T A V$ - $A T A A-L$, tricuspid aortic valve ascending thoracic aortic aneurysm circumferential region with respect to left coronary sinus. 


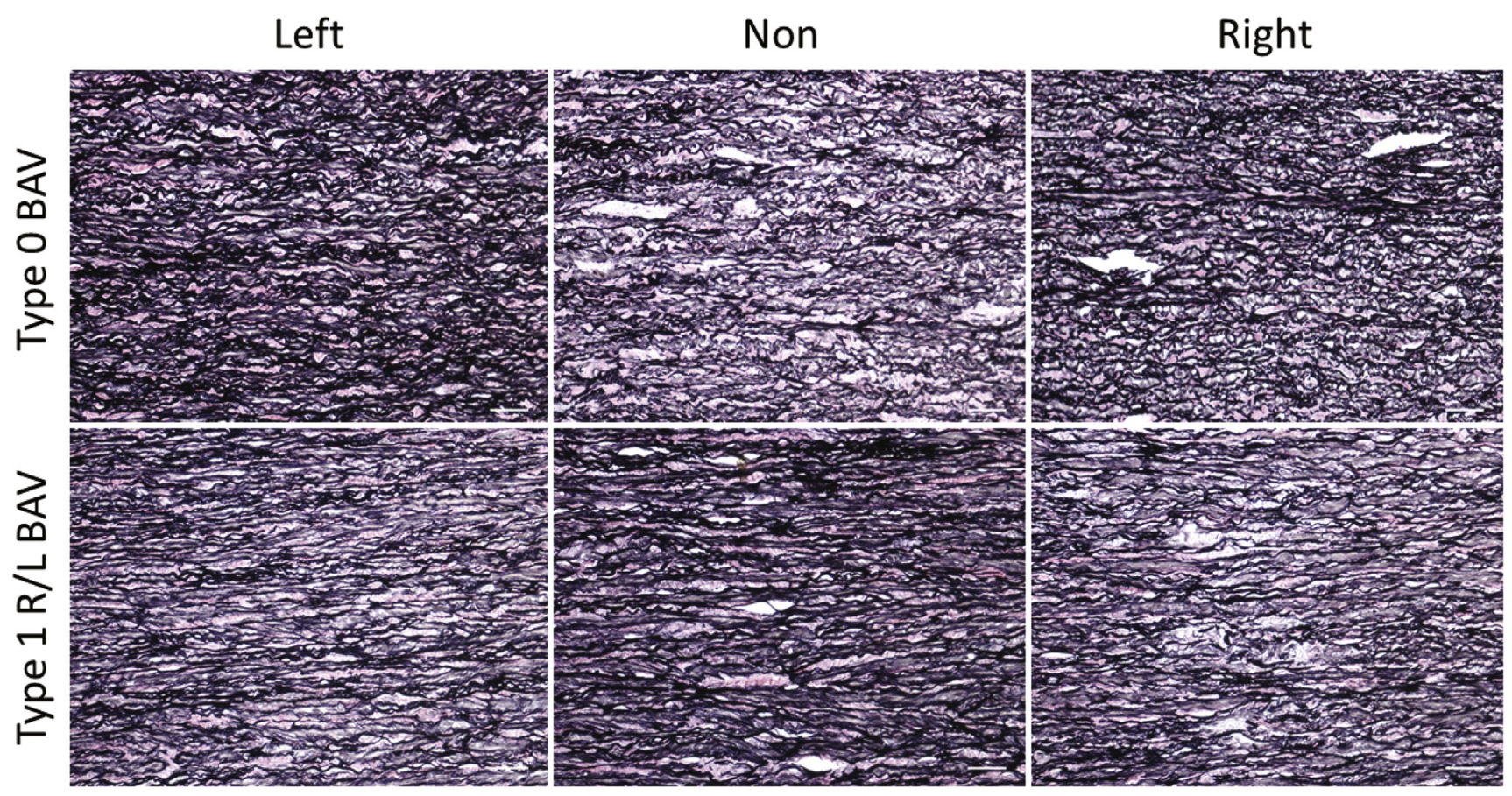

FIGURE E5. Human ascending thoracic aortic specimens from 3 circumferential regions with respect to proximity to the aortic valve cusps (left coronary [Left], noncoronary [Non], and right coronary [Right]) from 2 bicuspid aortic valve (BAV) patients displaying the 2 most common morphotypes (type 0 , top row, and type 1 right/left, bottom row). Five micron sections of formalin-fixed and paraffin-embedded specimens were evaluated for elastin composition and integrity using Verhoff-Van Gieson's stain. Specimens were visualized using bright field microscopy and a 20× objective. More extensive cystic medial degeneration was noted for regions Non and Right for BAV patients exhibiting both morphotypes. Scale bars $=50 \mu \mathrm{m}$. 